\title{
Synthesis of the Azepinobisindole Alkaloid Iheyamine A Enabled by a Cross-Mannich Reaction
}

Ashley C. Lindsay, Ivanhoe K. H. Leung and Jonathan Sperry*

j.sperry@auckland.ac.nz

\section{SUPPORTING INFORMATION}




\section{Table of Contents}

General Experimental $\quad$ S3

Experimental Procedures $\quad$ S4

Table S1 Comparison of Natural vs. Synthetic Iheyamine A

(Free base, ${ }^{1} \mathrm{H}$ NMR only)

S10

Table S2 Comparison of Natural vs. Synthetic Iheyamine A

(TFA salt; ${ }^{1} \mathrm{H}$ and ${ }^{13} \mathrm{C}$ NMR)

S11

${ }^{1} \mathrm{H}$ NMR spectrum of $\mathbf{6}$

S12

${ }^{13} \mathrm{C}$ NMR spectrum of 6

S13

${ }^{1} \mathrm{H}$ NMR spectrum of $7 \quad \mathrm{S14}$

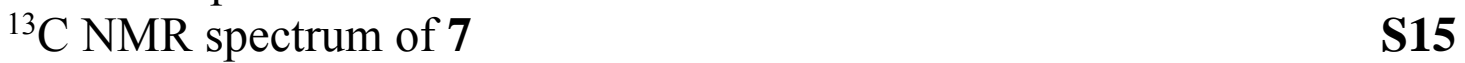

${ }^{1} \mathrm{H}$ NMR spectrum of $8 \quad$ S16

${ }^{13} \mathrm{C}$ NMR spectrum of $8 \quad \mathrm{S17}$

HMBC spectrum of $8 \quad$ S18

${ }^{1} \mathrm{H}$ NMR spectrum of $\mathbf{1 6} \quad \mathbf{S 1 9}$

${ }^{13} \mathrm{C}$ NMR spectrum of $\mathbf{1 6} \quad$ S20

NOESY spectrum of $\mathbf{1 6} \quad$ S21

${ }^{1} \mathrm{H}$ NMR spectrum of $\mathbf{1 7} \quad$ S22

${ }^{13}$ C NMR spectrum of $\mathbf{1 7} \quad$ S23

NOESY spectrum of $\mathbf{1 7} \quad$ S24

${ }^{1} \mathrm{H}$ NMR spectrum of $\mathbf{1 8} \quad$ S25

${ }^{13} \mathrm{C}$ NMR spectrum of $18 \quad$ S26

HMBC spectrum of $\mathbf{1 8} \quad$ S27

${ }^{1}$ H NMR of Synthetic Iheyamine A (free base) $\quad$ S28

Zoomed in ${ }^{1} \mathrm{H}$ NMR of Synthetic Iheyamine A (free base) $\quad$ S29

${ }^{1} \mathrm{H}$ NMR of Natural Iheyamine A (free base) $\quad$ S30

${ }^{1}$ H NMR of Synthetic Iheyamine A (TFA salt) $\quad$ S31

Zoomed in ${ }^{1} \mathrm{H}$ NMR of Synthetic Iheyamine A (TFA salt) $\quad$ S32

${ }^{1} \mathrm{H}$ NMR of Natural Iheyamine A (TFA salt) $\quad$ S33

${ }^{13}$ C NMR of Synthetic Iheyamine A (TFA salt) $\quad$ S34

Zoomed in ${ }^{13} \mathrm{C}$ NMR of Synthetic Iheyamine A (TFA salt)

${ }^{13} \mathrm{C}$ NMR of Natural Iheyamine A (TFA salt) $\quad$ S36

NOESY spectrum of Synthetic Iheyamine A $\quad$ S37

Variable Temperature ${ }^{1} \mathrm{H}$ NMR Experiments

For Iheyamine A (TFA salt) $\quad$ S38 


\section{Experimental Section}

\section{General}

All reactions were carried out in oven-dried glassware under a nitrogen atmosphere unless otherwise stated. Analytical thin layer chromatography was performed using $0.2 \mathrm{~mm}$ silica plates and compounds were visualized under $365 \mathrm{~nm}$ ultraviolet irradiation followed by staining with either alkaline permanganate or ethanolic vanillin solution. Infrared spectra were obtained as thin films between sodium chloride plates. Absorption maxima are expressed in wavenumbers $\left(\mathrm{cm}^{-1}\right)$. Melting points were recorded on a melting point apparatus and are uncorrected. NMR spectra were recorded as indicated on an NMR spectrometer operating at 500, 400 and $300 \mathrm{MHz}$ for ${ }^{1} \mathrm{H}$ nuclei and 125,100 and $75 \mathrm{MHz}$ for ${ }^{13} \mathrm{C}$ nuclei. Chemical shifts are reported in parts per million (ppm) relative to the tetramethylsilane peak recorded as $\delta 0.00$ ppm in $\mathrm{CDCl}_{3} / \mathrm{TMS}$ solvent, or the residual acetone ( $\left.\delta 2.05 \mathrm{ppm}\right)$, chloroform ( $\left.\delta 7.24 \mathrm{ppm}\right)$, DMSO ( $\delta 2.50 \mathrm{ppm})$ or methanol $(\delta 3.31 \mathrm{ppm})$ peaks. The ${ }^{13} \mathrm{C}$ NMR values were referenced to the residual acetone ( $\delta 29.9 \mathrm{ppm})$ chloroform $(\delta 77.1 \mathrm{ppm})$, DMSO $(\delta 39.5 \mathrm{ppm})$ or methanol ( $\delta 49.0$ ppm) peaks. ${ }^{13} \mathrm{C}$ NMR values are reported as chemical shift $\delta$ and assignment. ${ }^{1} \mathrm{H}$ NMR shift values are reported as chemical shift $\delta$, relative integral, multiplicity (s, singlet; d, doublet; t, triplet; q, quartet; m, multiplet), coupling constant ( $\mathrm{J}$ in $\mathrm{Hz}$ ) and assignment. Assignments are made with the aid of DEPT 90, DEPT 135, COSY, NOESY and HSQC experiments. ${ }^{1} \mathrm{H}-$ ${ }^{15} \mathrm{~N}$ HMBC NMR experiments (Bruker pulse programme hmbcf3gpndqf) were conducted at a ${ }^{1} \mathrm{H}$ frequency of $600 \mathrm{MHz}$ using a Bruker Avance spectrometer equipped with a TCI inverse cryoprobe. All experiments were conducted at $298 \mathrm{~K}$. Conventional NMR tubes $(5 \mathrm{~mm}$ diameter, Norell) using a sample volume of $500 \mu \mathrm{L}$ were used. Experiments using $J(\mathrm{XH})=5$ $\mathrm{Hz}$ and $10 \mathrm{~Hz}$ were conducted but in both cases no $1 \mathrm{H}-15 \mathrm{~N}$ correlations between the two $\mathrm{NH}$ tautomers were observed. ${ }^{1} \mathrm{H}$ VT Experiments were conducted at a ${ }^{1} \mathrm{H}$ frequency of $500 \mathrm{MHz}$ using a Bruker Avance III HD spectrometer equipped with a BBFO probe. Conventional NMR tubes ( $5 \mathrm{~mm}$ diameter, Norell) using a sample volume of $500 \mu \mathrm{L}$ were used. High resolution mass spectra were obtained by electrospray ionization in positive ion mode at a nominal accelerating voltage of $70 \mathrm{eV}$ on a microTOF mass spectrometer. 


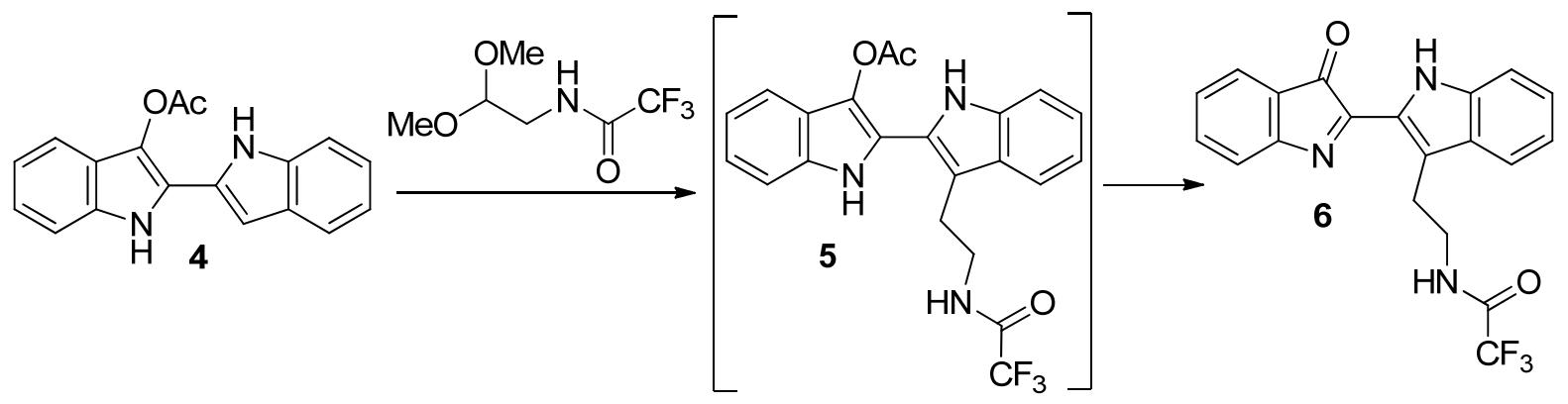

To a stirred solution of TFA $(0.34 \mathrm{~mL})$ and triethylsilane $(0.36 \mathrm{~mL})$ in dichloromethane $(4 \mathrm{~mL})$ was added a solution of bisindole $4^{1}(200 \mathrm{mg}, 0.68 \mathrm{mmol})$ in dichloromethane $(4 \mathrm{~mL})$ followed by $N$-(2,2-dimethoxyethyl)trifluoroacetamide ${ }^{2}(156 \mathrm{mg}, 0.78 \mathrm{mmol})$ in dichloromethane (4 $\mathrm{mL}$ ) and the resulting solution was stirred at $50{ }^{\circ} \mathrm{C}$ for $1.5 \mathrm{~h}$. The reaction was neutralised with sodium bicarbonate (sat. sol, $50 \mathrm{~mL})$ and the mixture was extracted with dichloromethane $(3 \mathrm{x}$ $20 \mathrm{~mL})$. The organic extracts were combined, dried $\left(\mathrm{Na}_{2} \mathrm{SO}_{4}\right)$, filtered and concentrated under reduced pressure to give the unstable tryptamine 5 which was immediately solubilised in methanol (10 mL) and water (2 mL). Potassium carbonate $(290 \mathrm{mg}, 2.1 \mathrm{mmol})$ was added and the mixture stirred at room temperature in an open flask for 5 min. Ethyl acetate $(50 \mathrm{~mL})$ was added and the mixture was washed with brine $(3 \times 50 \mathrm{~mL})$. The organic layer was dried ( $\mathrm{Na}_{2} \mathrm{SO}_{4}$ ), filtered and concentrated in vacuo. Purification of the crude solid by flash chromatography on silica gel eluting with hexanes-ethyl acetate (3:1) gave the title compound (167 mg, $0.40 \mathrm{mmol}, 59 \%$ ) as a blue solid, M.p. $173.0-175.0{ }^{\circ} \mathrm{C}$; HRMS [ESI, $(\mathrm{M}+\mathrm{Na})^{+}$] found $408.0940\left[\mathrm{C}_{20} \mathrm{H}_{14} \mathrm{~F}_{3} \mathrm{~N}_{3} \mathrm{O}_{2}+\mathrm{Na}\right]^{+}$requires 408.0930; $v_{\max } / \mathrm{cm}^{-1}$ (neat) 3382, 1714, 1701, 1147, 3062; $\delta_{\mathrm{H}}\left(400 \mathrm{MHz},\left(\mathrm{CD}_{3}\right)_{2} \mathrm{SO}\right) 11.40(1 \mathrm{H}$, br s, NH), $9.55(1 \mathrm{H}$, br t, J 5.0, NH), 7.71$7.61(4 \mathrm{H}, \mathrm{m}, 4 \mathrm{x} \mathrm{CH}), 7.43(1 \mathrm{H}, \mathrm{d}, J$ 7.6, CH), 7.33 (1 H, t, J 7.6, CH), $7.28(1 \mathrm{H}, \mathrm{t}, J$ 7.6, $\mathrm{CH}), 7.09$ (1 H, t, J 7.6, CH), 3.61-3.56 (2 H, m, CH 2$), 3.51-3.48\left(2 \mathrm{H}, \mathrm{m}, \mathrm{CH}_{2}\right) ; \delta_{\mathrm{C}}(100 \mathrm{MHz}$, $\left.\left(\mathrm{CD}_{3}\right)_{2} \mathrm{SO}\right) 193.5(\mathrm{C}), 161.0(\mathrm{C}), 155.7(\mathrm{C}), 138.4(\mathrm{C}), 137.3(\mathrm{CH}), 128.0(\mathrm{C}), 127.7(\mathrm{CH})$, $125.3(\mathrm{CH}), 125.0(\mathrm{C}), 124.7(\mathrm{CH}), 121.9(2 \times \mathrm{C}), 121.4(\mathrm{CH}), 119.9(\mathrm{CH}), 119.7(\mathrm{CH}), 113.0$ $(\mathrm{CH}), 39.9\left(\mathrm{CH}_{2}\right), 24.2\left(\mathrm{CH}_{2}\right), 2 \times \mathrm{C}$ not observed.

1. McErlean, C. S. P.; Sperry, J.; Blake. A. J.; Moody, C. J. Tetrahedron 2007, 63, 10963.

2. Righi, M,; Bedini, A.; Piersanti, G.; Romagnoli, F.; Spadoni, G. J. Org. Chem. 2011, 76, 704. 


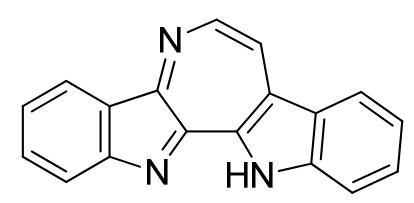

To a solution of bisindole $6(166 \mathrm{mg}, 0.43 \mathrm{mmol})$ in methanol $(10 \mathrm{~mL})$ and water $(2 \mathrm{~mL})$ was added potassium carbonate $(200 \mathrm{mg}, 1.5 \mathrm{mmol})$ and the reaction mixture stirred at $110^{\circ} \mathrm{C}$ for $30 \mathrm{~min}$. After cooling to room temperature, water $(20 \mathrm{~mL})$ was added and the whole extracted with ethyl acetate $(3 \times 20 \mathrm{~mL})$. The combined organic extracts were dried $\left(\mathrm{Na}_{2} \mathrm{SO}_{4}\right)$, filtered and concentrated in vacuo. Purification by flash chromatography on silica gel eluting with hexanes-ethyl acetate (3:2) gave the title compound (20 mg, $0.07 \mathrm{mmol}, 18 \%$ ) as a purple solid, M.p. $278.0-281.3{ }^{\circ} \mathrm{C}$ (charred); HRMS [ESI, $\left.(\mathrm{M}+\mathrm{H})^{+}\right]$found $270.1029\left[\mathrm{C}_{18} \mathrm{H}_{11} \mathrm{~N}_{3}+\mathrm{H}\right]^{+}$ requires 270.1026; $\nu_{\max } / \mathrm{cm}^{-1}$ (neat) $2640,1588,1381,1183,1030 ; \delta_{\mathrm{H}}\left(400 \mathrm{MHz}, \mathrm{CDCl}_{3}\right) 9.10$ (1 H, d, J 6.6, CH), 8.61-8.58 (2 H, m, 2 x CH), 8.42 (1 H, d, J 8.1, CH), 7.73 (1 H, d, J 8.1, $\mathrm{CH}), 7.67(1 \mathrm{H}, \mathrm{t}, J$ 7.5, CH), $7.61(1 \mathrm{H}, \mathrm{t}, J$ 7.5, CH), 7.74-7.48 (3 H, m, 3 x CH), 1 x NH not observed; $\delta_{\mathrm{C}}\left(100 \mathrm{MHz}, \mathrm{CDCl}_{3}\right) 151.2(\mathrm{C}), 146.4(\mathrm{C}), 146.2(\mathrm{C}), 145.4(\mathrm{C}), 140.6(\mathrm{CH}), 136.9$ (C), $135.1(\mathrm{C}), 130.7(\mathrm{CH}), 130.0(\mathrm{CH}), 127.3(\mathrm{C}), 126.6(\mathrm{C}), 122.8(\mathrm{CH}), 122.6(\mathrm{CH}), 122.0$ $(\mathrm{CH}), 121.9(\mathrm{CH}), 121.3(\mathrm{CH}), 117.3(\mathrm{CH}), 115.0(\mathrm{CH})$.

\section{2,2,2-Trifluoro- $N$-(2-(5'-methoxy-[2,2'-bisindol]-3-yl)ethyl)acetamide (8)}

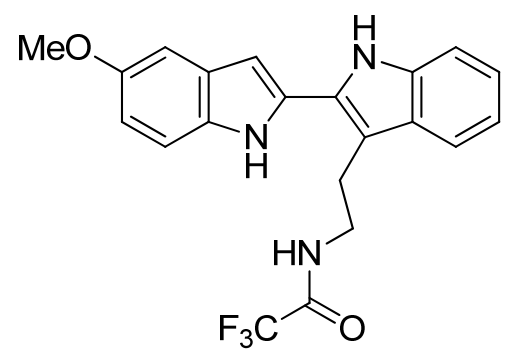

To a solution of tryptamine $11^{3}(10 \mathrm{mg}, 0.039 \mathrm{mmol})$ in trifluoroacetic acid $(0.5 \mathrm{~mL})$ at $0{ }^{\circ} \mathrm{C}$ was added 3-acetoxy-5-methoxyindole $15^{4}(24 \mathrm{mg}, 0.12 \mathrm{mmol})$ in dichloromethane $(0.1 \mathrm{~mL})$ dropwise over $5 \mathrm{~min}$. The reaction mixture was stirred at $0{ }^{\circ} \mathrm{C}$ for $5 \mathrm{~min}$, dichloromethane (5 $\mathrm{mL}$ ) was added and the organic layer was washed carefully with sodium bicarbonate (sat. sol, $3 \times 20 \mathrm{~mL})$. The organic extract was dried $\left(\mathrm{Na}_{2} \mathrm{SO}_{4}\right)$, filtered and concentrated in vacuo. Purification by flash chromatography on silica gel eluting with toluene-acetone $(9: 1)$ gave the title compound (14 mg, $0.034 \mathrm{mmol}, 93 \%$ ) as a colourless solid. M.p. $188.3-190.2{ }^{\circ} \mathrm{C}$ (charred); HRMS [ESI, $\left.(\mathrm{M}+\mathrm{Na})^{+}\right]$found $424.1241\left[\mathrm{C}_{21} \mathrm{H}_{18} \mathrm{~F}_{3} \mathrm{~N}_{3} \mathrm{O}_{2}+\mathrm{Na}\right]^{+}$requires 424.1243; 
$V_{\max } / \mathrm{cm}^{-1}$ (neat) $3339,1703,1446,1341,1142 ; \delta_{\mathrm{H}}\left(400 \mathrm{MHz},\left(\mathrm{CD}_{3}\right)_{2} \mathrm{SO}\right) 11.17(1 \mathrm{H}, \mathrm{br} \mathrm{s}, \mathrm{NH})$, 11.04 (1 H, br s, NH), $9.69(1 \mathrm{H}$, br t, $J$ 5.0, NH), $7.60(1 \mathrm{H}, \mathrm{d}, J$ 8.0, CH), $7.41(1 \mathrm{H}, \mathrm{d}, J$ 8.0, CH), 7.37 (1 H, d, J 8.5, CH), $7.15(1 \mathrm{H}, \mathrm{t}, J$ 7.1, CH), 7.08-7.05 (2 H, m, 2 x CH), 6.81-6.79 $(2 \mathrm{H}, \mathrm{m}, 2 \mathrm{x} \mathrm{CH}), 3.80(3 \mathrm{H}, \mathrm{s}, \mathrm{Me}), 3.50-3.45\left(2 \mathrm{H}, \mathrm{m}, \mathrm{CH}_{2}\right), 3.21\left(2 \mathrm{H}, \mathrm{t}, J \mathrm{~J} .6, \mathrm{CH}_{2}\right)$; $\delta_{\mathrm{C}}$ (100MHz, (CD $\left.)_{2} \mathrm{SO}\right) 153.8$ (C), 135.9 (C), 131.7 (C), 130.9 (C), 128.9 (C), 128.7 (C), 127.9 (C), $121.9(\mathrm{CH}), 119.1(\mathrm{CH}), 118.1(\mathrm{CH}), 112.0(\mathrm{CH}), 111.9(\mathrm{CH}), 111.1(\mathrm{CH}), 108.8(\mathrm{C})$, $101.4(\mathrm{CH}), 100.5(\mathrm{CH}), 55.3(\mathrm{Me}), 38.9\left(\mathrm{CH}_{2}\right), 24.0\left(\mathrm{CH}_{2}\right), 2$ x C not observed.

The two homodimers 16 and 17 were also isolated (7 mg combined mass). Characterisation data as follows:

5,5'-Dimethoxy-[2,2'-bisindol]-3-yl acetate (16)<smiles>COc1ccc2[nH]c(-c3[nH]c4ccc(OC)cc4c3OC)cc2c1</smiles>

(2 mg, $0.006 \mathrm{mmol})$; M.p. $168.1-171.2^{\circ} \mathrm{C}$ (charred); HRMS [ESI, $(\mathrm{M}+\mathrm{Na})^{+}$] found 373.1152 $\left[\mathrm{C}_{20} \mathrm{H}_{18} \mathrm{~N}_{2} \mathrm{O}_{4}+\mathrm{Na}\right]^{+}$requires $373.1159 ; v_{\max } / \mathrm{cm}^{-1}$ (neat) $3404,3327,2958,1737,1706 ; \delta_{\mathrm{H}}(400$ MHz, (CD $\left.)_{2} \mathrm{SO}\right) 11.14(1 \mathrm{H}$, br s, NH), 10.91 (1 H, br s, NH), 7.37 (1 H, d, J 8.8, CH), 7.32 (1 H, d, J 9.7, CH), 7.09 (1 H, d, J 2.3, CH), $6.82-6.77$ (3 H, m, 3 x CH), 6.73 (1 H, d, J 1.4, $\mathrm{CH}), 3.79$ (3 H, s, Me), 3.78 (3 H, s, Me), 2.50 (3 H, s, Me); $\delta_{\mathrm{C}}\left(100 \mathrm{MHz},\left(\mathrm{CD}_{3}\right)_{2} \mathrm{SO}\right) 169.2$ (C), 153.8 (C), 153.7 (C), 131.8 (C), 129.1 (C), 128.7 (C), 128.6 (C), 125.6 (C), 121.5 (C), $120.9(\mathrm{C}), 112.8(\mathrm{CH}), 112.5(\mathrm{CH}), 112.2(\mathrm{CH}), 112.1(\mathrm{CH}), 101.4(\mathrm{CH}), 99.7(\mathrm{CH}), 98.7(\mathrm{CH})$, $55.4(\mathrm{Me}), 55.3(\mathrm{Me}), 20.9(\mathrm{Me})$.

\section{5,5'-Dimethoxy-[2,4'-bisindol]-3'-yl acetate (17)}<smiles>COc1ccc2[nH]c(-c3c(OC)ccc4[nH]cc(OC(C)=O)c34)cc2c1</smiles>

(5 mg, 0.014 mmol); M.p. $141.3-147.2{ }^{\circ} \mathrm{C}$; HRMS [ESI, $(\mathrm{M}+\mathrm{Na})^{+}$] found 373.1152 $\left[\mathrm{C}_{20} \mathrm{H}_{18} \mathrm{~N}_{2} \mathrm{O}_{4}+\mathrm{Na}\right]^{+}$requires $373.1159 ; v_{\max } / \mathrm{cm}^{-1}$ (neat) $3402,3333,2839,1738,1705 ; \delta_{\mathrm{H}}(400$ 
MHz, (CD $\left.)_{2} \mathrm{SO}\right) 10.98(1 \mathrm{H}$, br s, NH), 10.83 (1 H, br s, NH), 7.41 (1 H, d, J 8.5, CH), 7.29 (1 H, d, J 2.8, CH), 7.27 (1 H, d, J 8.5, CH), 7.07-7.04 (2 H, m, 2 x CH), 6.71 (1 H, dd, J 9.0, 2.4, $\mathrm{CH}), 6.26(1 \mathrm{H}, \mathrm{d}, J$ 1.7, CH), $3.78(3 \mathrm{H}, \mathrm{s}, \mathrm{Me}), 3.73(3 \mathrm{H}, \mathrm{s}, \mathrm{Me}), 1.50(3 \mathrm{H}, \mathrm{s}, \mathrm{Me}) ; \delta \mathrm{c}$ (100MHz, (CD $\left.)_{2} \mathrm{SO}\right) 168.8(\mathrm{C}), 153.1$ (C), 151.1 (C), 132.8 (C), 131.3 (C), 129.8 (C), 128.6 (C), $128.2(\mathrm{C}), 118.9(\mathrm{C}), 117.4(\mathrm{CH}), 112.5(\mathrm{CH}), 111.51(\mathrm{C}), 111.47(\mathrm{CH}), 110.4(\mathrm{CH}), 109.4$ $(\mathrm{CH}), 102.3(\mathrm{CH}), 101.0(\mathrm{CH}), 57.2(\mathrm{Me}), 55.3(\mathrm{Me}), 19.8(\mathrm{Me})$.

3. Kexin, L.; Ping, W.; Jin, L.; Guosheng, H. Synthesis 2010, 21, 3623.

4. De la Fuente, M.; Dominguez, D. Tetrahedron 2011, 67, 3997.

\section{5,5'-Dimethoxy-2,3'-bisindole (14)}

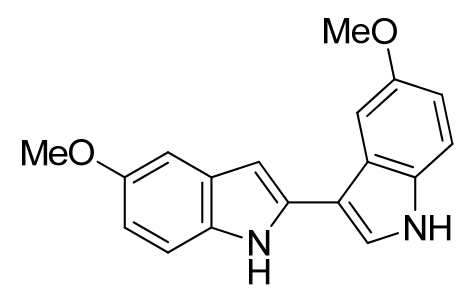

Obtained from the failed cross-Mannich reaction between indole $\mathbf{1 3}$ and tryptamine 11, spectroscopic data as follows:

(8 mg, $0.03 \mathrm{mmol}, 67 \%$ ) as a yellow oil. $\delta_{\mathrm{H}}\left(400 \mathrm{MHz},\left(\mathrm{CD}_{3}\right)_{2} \mathrm{SO}\right) 11.24(1 \mathrm{H}, \mathrm{br} \mathrm{s}, \mathrm{NH}), 10.99$ (1 H, br s, NH), $7.79(1 \mathrm{H}, \mathrm{d}, J$ 2.5, CH), $7.40(1 \mathrm{H}, \mathrm{d}, J$ 1.9, CH), $7.37(1 \mathrm{H}, \mathrm{d}, J$ 8.8, CH), 7.24 (1 H, d, J 8.5, CH), 7.04 (1 H, d, J 2.4, CH), 6.84 (1 H, dd, J 8.8, 2.5, CH), 6.69-6.67 (2 H, m, $2 \times \mathrm{CH}), 3.86(3 \mathrm{H}, \mathrm{s}, \mathrm{Me}), 3.78(3 \mathrm{H}, \mathrm{s}, \mathrm{Me})$; NMR is consistent with the literature. ${ }^{5}$

5. Guo, T.; Han, S.; Liu, Y.; Liu, Y.; Liu, H. Tetrahedron Lett. 2016, 57, 1097. 


\section{2,2,2-Trifluoro- $N$-(2-(5'-methoxy-3'-oxo-[2,2'-bisindol]-3-yl)ethyl)acetamide (18)}<smiles>COc1ccc2c(c1)C(=O)C(Cc1c(CCNC(=O)C(F)(F)F)[nH]c3ccccc13)=N2</smiles>

To a solution of bisindole $8(100 \mathrm{mg}, 0.24 \mathrm{mmol})$ in DMF $(6 \mathrm{~mL})$ was added potassium hydroxide $(25 \mathrm{mg}, 0.45 \mathrm{mmol})$ and iodine $(60 \mathrm{mg}, 0.24 \mathrm{mmol})$ and the reaction mixture stirred for $10 \mathrm{~min}$ at room temperature. The mixture was then purged with air, silica (100 $\mathrm{mg})$ was added and the mixture heated to $120^{\circ} \mathrm{C}$ for $20 \mathrm{~min}$. Upon cooling, water was added and the mixture extracted with ethyl acetate $(3 \times 20 \mathrm{~mL})$. The organic extracts were combined, dried $\left(\mathrm{Na}_{2} \mathrm{SO}_{4}\right)$, filtered and concentrated in vacuo. Purification by flash chromatography on silica get eluting with hexanes-acetone (2:1) gave the title compound ( $87 \mathrm{mg}, 0.21 \mathrm{mmol}, 87 \%$ ) as a blue solid, M.p. 209.1 - $212.9{ }^{\circ} \mathrm{C}$ (charred); HRMS [ESI, $(\mathrm{M}+\mathrm{Na})^{+}$] found 438.1035 $\left[\mathrm{C}_{21} \mathrm{H}_{16} \mathrm{~F}_{3} \mathrm{~N}_{3} \mathrm{O}_{3}+\mathrm{Na}\right]^{+}$requires $438.1036 ; v_{\max } / \mathrm{cm}^{-1}$ (neat): 3382, 3059, 1714, 1519, 1477; $\delta_{\mathrm{H}}$ (400 MHz, (CD $\left.)_{2} \mathrm{SO}\right) 11.33(1 \mathrm{H}$, br s, NH), 9.55 (1 H, t, J 5.5, NH), 7.68 (1 H, d, J 8.1, CH), $7.61(1 \mathrm{H}, \mathrm{d}, J$ 8.1, CH), 7.35 (1 H, d, J 8.1, CH), 7.26 (1 H, t, J 7.6, CH), $7.22(1 \mathrm{H}, \mathrm{d}, J$ 2.5, $\mathrm{CH}), 7.16$ (1 H, dd, J 8.1, 2.5, CH), 7.08 (1 H, t, J 7.6, CH), $3.86(3 \mathrm{H}, \mathrm{s}, \mathrm{Me}), 3.59-3.55$ (2 H, m, $\left.\mathrm{CH}_{2}\right), 3.48-3.45\left(2 \mathrm{H}, \mathrm{m}, \mathrm{CH}_{2}\right)$; $\delta_{\mathrm{C}}\left(100 \mathrm{MHz},\left(\mathrm{CD}_{3}\right)_{2} \mathrm{SO}\right) 193.4(\mathrm{C}), 159.3(\mathrm{C}), 156.2$ (d, J 35.4, C), 154.7 (C), 153.7 (C), 138.1 (C), 128.0 (C), 125.1 (C), 124.9 (CH), 123.0 (C), 122.2 $(\mathrm{CH}), 120.8(\mathrm{CH}), 120.6(\mathrm{C}), 119.8(\mathrm{CH}), 119.6(\mathrm{CH}), 112.9(\mathrm{CH}), 111.2(\mathrm{CH}), 156.0(\mathrm{Me})$, $39.9\left(\mathrm{CH}_{2}\right), 24.1\left(\mathrm{CH}_{2}\right), 1 \times \mathrm{C}$ not observed. 


\section{Iheyamine A (1; Free base)}<smiles>COc1ccc2nc3c4[nH]c5ccccc5c4ccnc-3c2c1</smiles>

To a solution of bisindole $18(23 \mathrm{mg}, 0.055 \mathrm{mmol})$ in methanol $(3 \mathrm{~mL})$ and water $(0.5 \mathrm{~mL})$ was added potassium carbonate $(100 \mathrm{mg}, 0.31 \mathrm{mmol})$ and the reaction mixture stirred at $110{ }^{\circ} \mathrm{C}$ for $30 \mathrm{~min}$. Ethyl acetate $(5 \mathrm{~mL})$ was added and the whole was washed with brine $(3 \mathrm{x} 5 \mathrm{~mL})$. The organic layer was dried $\left(\mathrm{Na}_{2} \mathrm{SO}_{4}\right)$, filtered and concentrated in vacuo. Purification by flash chromatography on silica gel eluting with dichloromethane:methanol (9:1) gave the title compound (11 mg, $0.037 \mathrm{mmol}, 69 \%$ ) as a purple solid, M.p. $290-295{ }^{\circ} \mathrm{C}$ (charred); HRMS [ESI, $\left.(\mathrm{M}+\mathrm{H})^{+}\right]$found $300.1131\left[\mathrm{C}_{19} \mathrm{H}_{13} \mathrm{~N}_{3} \mathrm{O}+\mathrm{H}\right]^{+}$requires $300.1131 ; v_{\max } / \mathrm{cm}^{-1}$ (neat): 2637 , 1620, 1583, 1484, 1381.

Personal communication with Professor Junichi Tanaka revealed that the free base of iheyamine $\mathrm{A}$ in very insoluble, hence only a ${ }^{1} \mathrm{H}$ NMR could be obtained. We also found this to be the case with the synthetic sample; our ${ }^{1} \mathrm{H}$ NMR spectrum matched that of the natural product (Table S1). For full characterisation, the isolation authors characterised the TFA salt of iheymane A. ${ }^{6}$ Our synthetic sample of iheyamine A was converted to the TFA salt as follows:

\section{Iheyamine A (1; TFA salt)}<smiles>COc1ccc2nc3c4[nH]c5ccc(C(F)(F)F)cc5c4ccnc-3c2c1</smiles>

To iheyamine A free base $(5 \mathrm{mg}, 0.016 \mathrm{mmol})$ in $\mathrm{CDCl}_{3}(1.5 \mathrm{~mL})$ was added TFA (4 drops). The resulting solution stirred for 20 minutes at room temperature before being concentrated in vacuo to give the title compound (7 $\mathrm{mg}, 0.016 \mathrm{mmol}, 100 \%$ ) as a purple solid, M.p. 230-236 ${ }^{\circ} \mathrm{C}$ (charred); HRMS [ESI, $\left.(\mathrm{M}+\mathrm{H})^{+}\right]$found $300.1129\left[\mathrm{C}_{19} \mathrm{H}_{13} \mathrm{~N}_{3} \mathrm{O}+\mathrm{H}\right]^{+}$requires 300.1131 ; $v_{\max } / \mathrm{cm}^{-1}$ (neat): $3260,1620,1599,1392,1340,809,782$; NMR data see Table S2

6. Sasaki, T.; Ohtani, I.I.; Tanaka, J.; Higa, T. Tetrahedron. Lett. 1999, 40, 303. 
Table S1 ${ }^{1} \mathrm{H}$ NMR data for iheyamine A free base ${ }^{6}$ and the synthetic sample prepared herein

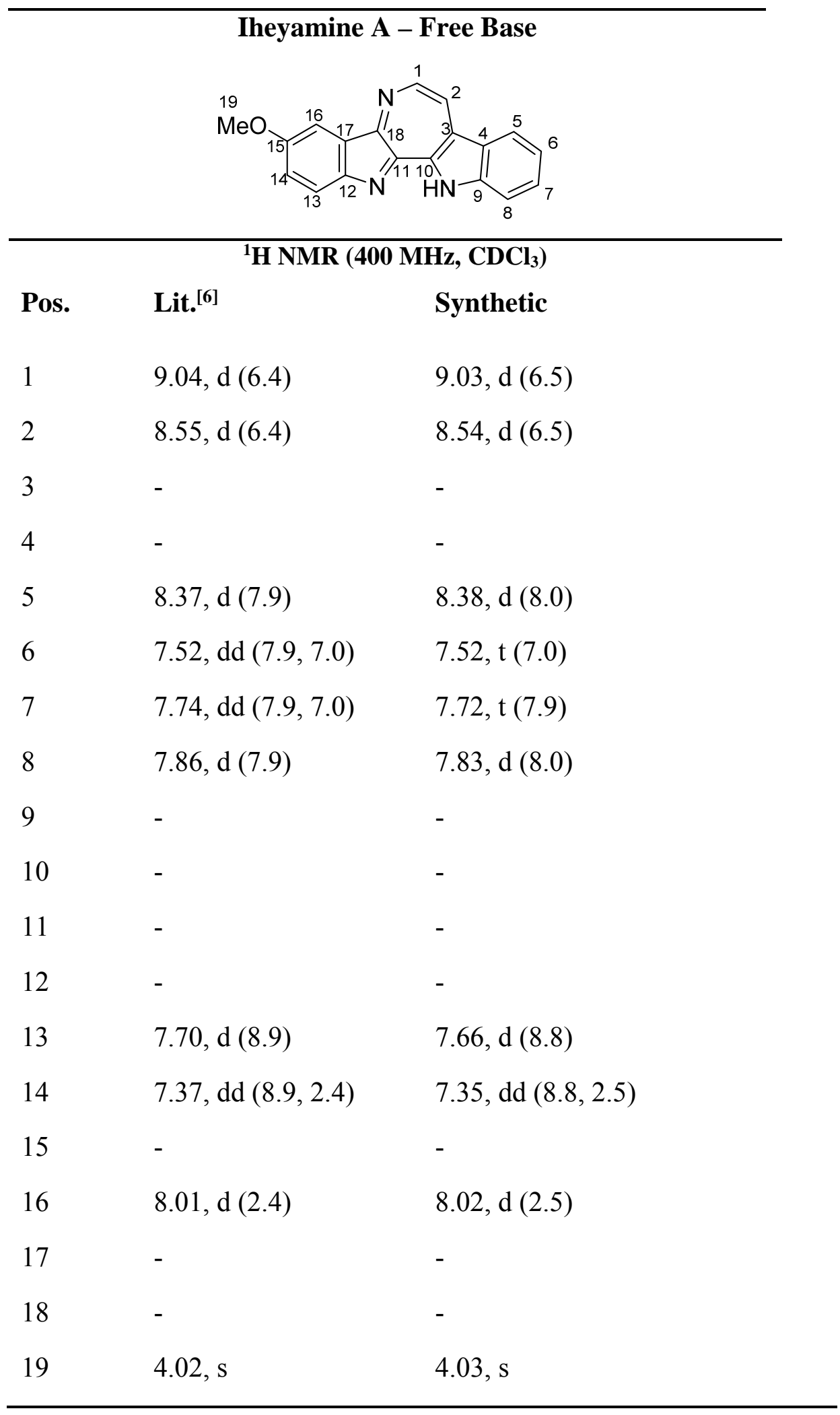


Table S2 ${ }^{1} \mathrm{H}$ and ${ }^{13} \mathrm{C}$ NMR data for iheyamine A TFA salt ${ }^{6}$ and the synthetic sample prepared herein

\begin{tabular}{|c|c|c|c|c|}
\hline \multicolumn{5}{|c|}{ Iheyamine A - TFA Salt } \\
\hline \multicolumn{3}{|c|}{${ }^{1} \mathrm{H}$ NMR (400MHz, $\left.\mathrm{CDCl}_{3}\right)$} & \multicolumn{2}{|c|}{${ }^{13} \mathrm{C}$ NMR (100MHz, $\left.\mathrm{CDCl}_{3}\right)$} \\
\hline \multicolumn{3}{|c|}{ ppm } & \multicolumn{2}{|c|}{ ppm } \\
\hline Pos. & Lit. ${ }^{6}$ & Synthetic & Lit. ${ }^{6}$ & Synthetic \\
\hline 1 & $9.38, \mathrm{~d}(6.4)$ & $9.38, \mathrm{~d}(6.5)$ & $143.2, \mathrm{CH}$ & $143.1, \mathrm{CH}$ \\
\hline 2 & $8.85, \mathrm{~d}(6.4)$ & $8.85, \mathrm{~d}(6.52)$ & $124.5, \mathrm{CH}$ & $124.5, \mathrm{CH}$ \\
\hline 3 & - & - & 128.3, C & $128.4, \mathrm{C}$ \\
\hline 4 & - & - & $123.8, \mathrm{C}$ & $123.9, \mathrm{C}$ \\
\hline 5 & $8.42, \mathrm{~d}(7.9)$ & $8.42, \mathrm{~d}(7.9)$ & $121.4, \mathrm{CH}$ & $121.4, \mathrm{CH}$ \\
\hline 6 & $7.61, \mathrm{dd}(7.9,7.0)$ & $7.61, \mathrm{t}(7.0)$ & $124.4, \mathrm{CH}$ & $124.4, \mathrm{CH}$ \\
\hline 7 & $7.84, \mathrm{dd}(7.9,7.0)$ & $7.85, \mathrm{t}(7.9)$ & $132.8, \mathrm{CH}$ & $132.8, \mathrm{CH}$ \\
\hline 8 & $8.05, \mathrm{~d}(7.9)$ & $8.06, \mathrm{~d}(8.0)$ & $115.1, \mathrm{CH}$ & $115.1, \mathrm{CH}$ \\
\hline 9 & - & - & $144.0, \mathrm{C}$ & 144.1, C \\
\hline 10 & - & - & $138.1, \mathrm{C}$ & $138.15, \mathrm{C}$ \\
\hline 11 & - & - & $148.9, \mathrm{C}$ & $148.9, \mathrm{C}$ \\
\hline 12 & - & - & $138.2, \mathrm{C}$ & $138.23, \mathrm{C}$ \\
\hline 13 & $7.99, \mathrm{~d}(8.9)$ & $7.99, \mathrm{~d}(8.9)$ & $115.7, \mathrm{CH}$ & $115.8, \mathrm{CH}$ \\
\hline 14 & $7.52, \mathrm{dd}(8.9,2.4)$ & $7.52, \mathrm{dd}(8.9,2.52)$ & 124.1, CH & 124.1, CH \\
\hline 15 & - & - & $157.5, \mathrm{C}$ & $157.5, \mathrm{C}$ \\
\hline 16 & $7.96, \mathrm{~d}(2.4)$ & $7.97, \mathrm{~d}(2.5)$ & $102.8, \mathrm{CH}$ & $102.7, \mathrm{CH}$ \\
\hline 17 & - & - & 126.1, C & 126.1, C \\
\hline 18 & - & - & $135.7, \mathrm{C}$ & $135.7, \mathrm{C}$ \\
\hline 19 & $4.02, \mathrm{~s}$ & $4.02, \mathrm{~s}$ & $56.0, \mathrm{Me}$ & $56.0, \mathrm{Me}$ \\
\hline
\end{tabular}




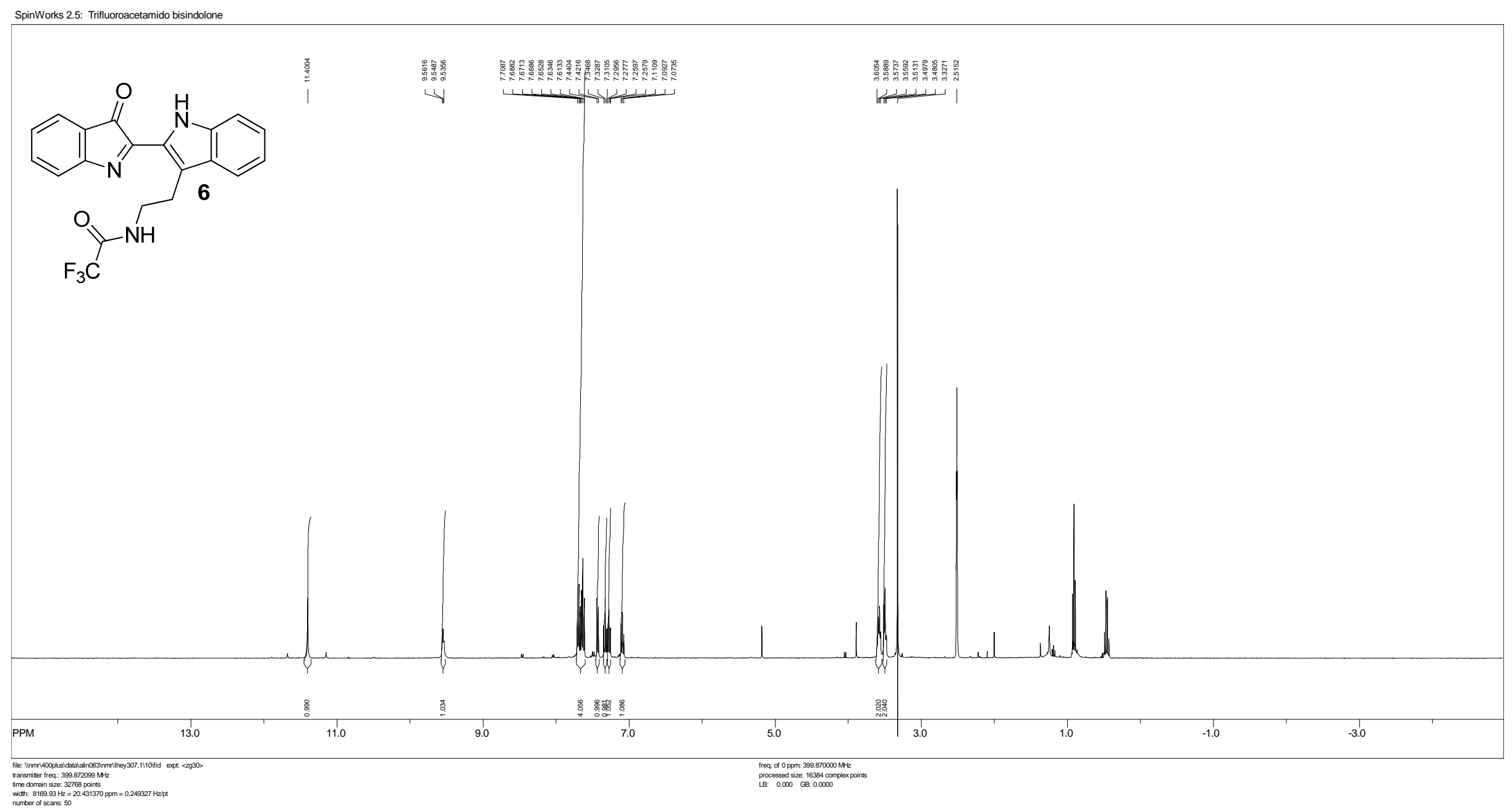

12 


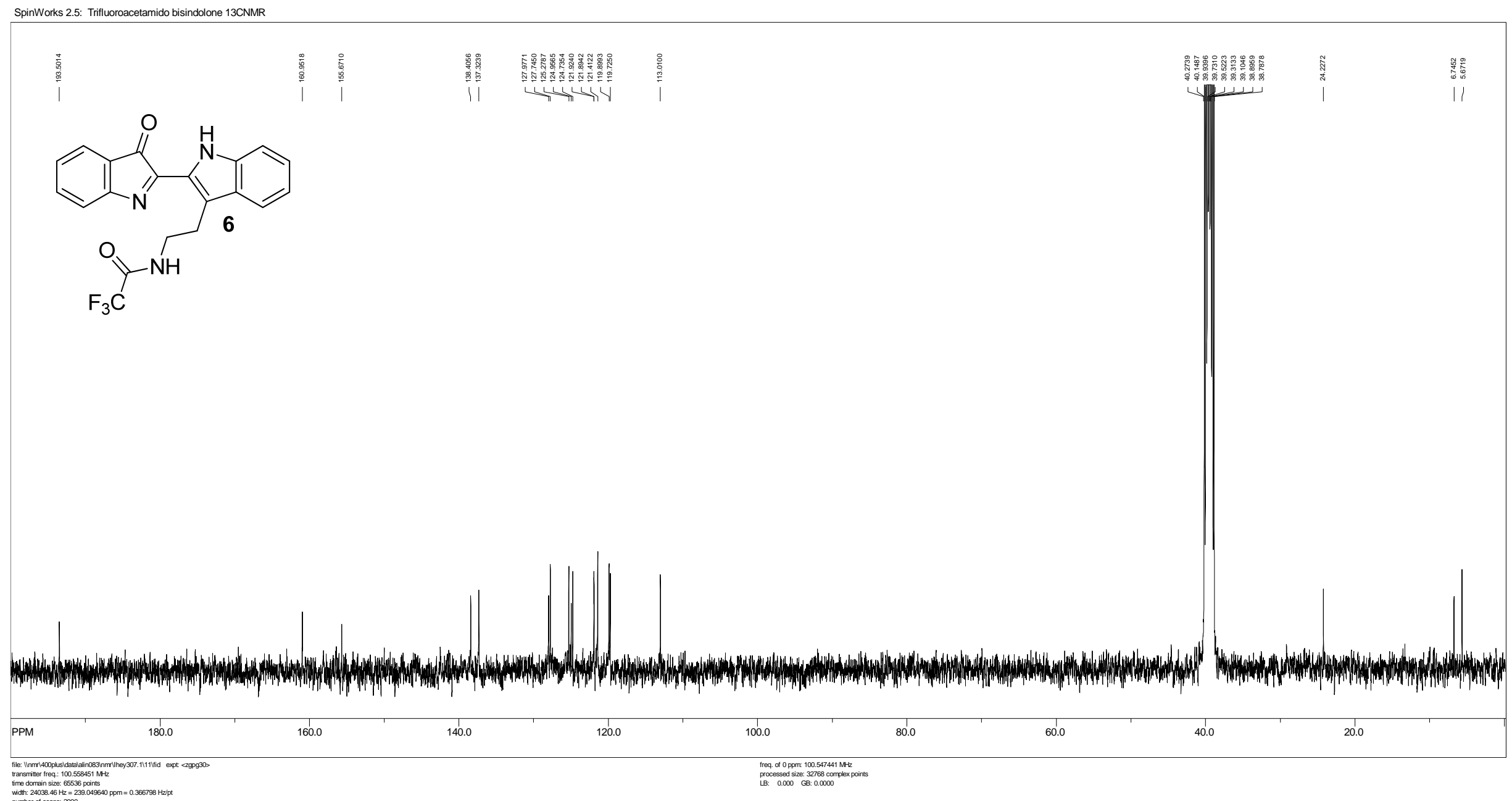


SpinWorks 2.5: Ineyamine A Core

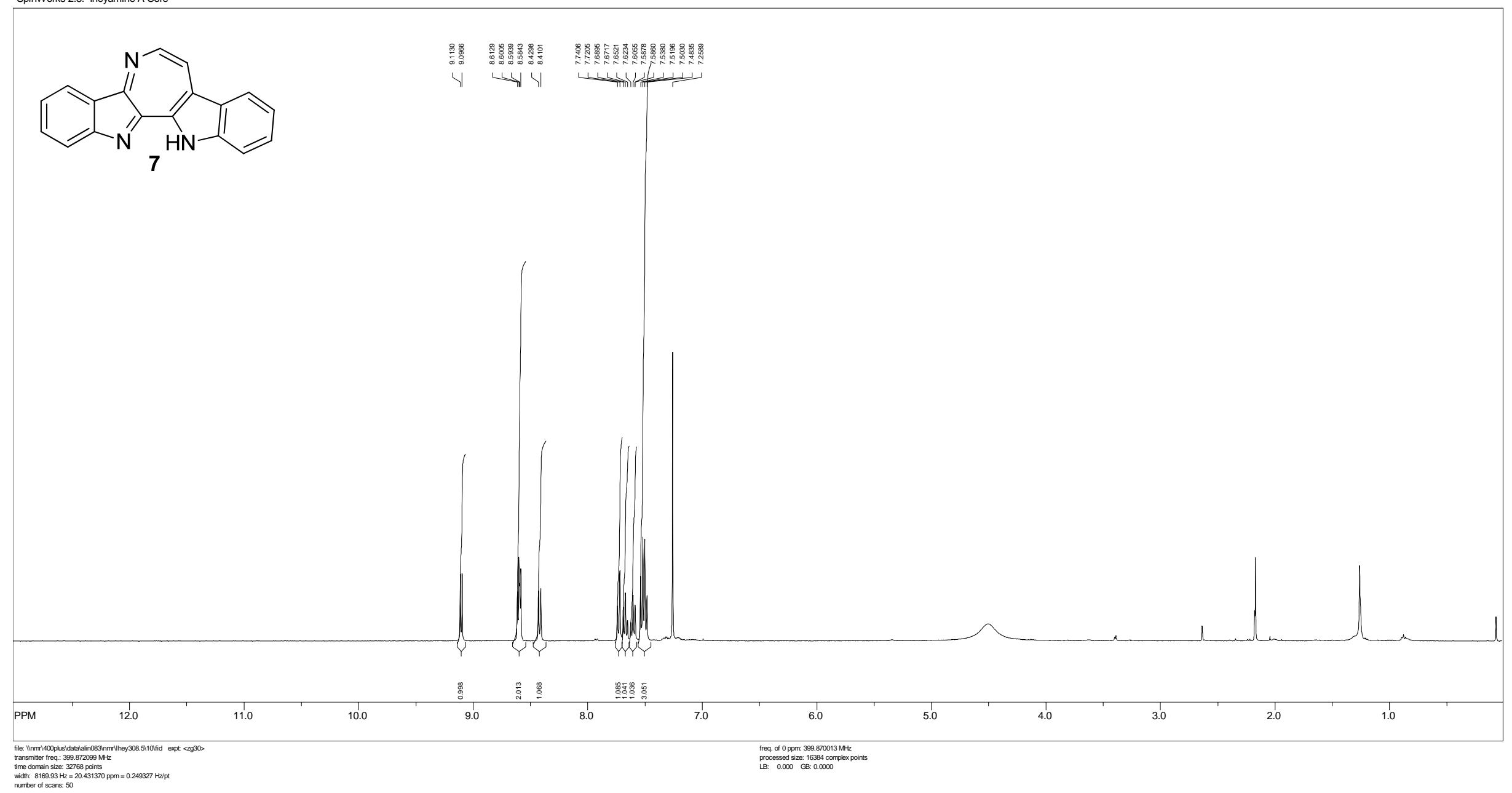


SpinWorks 2.5: Iheyamine A Core Structure 13C NMR

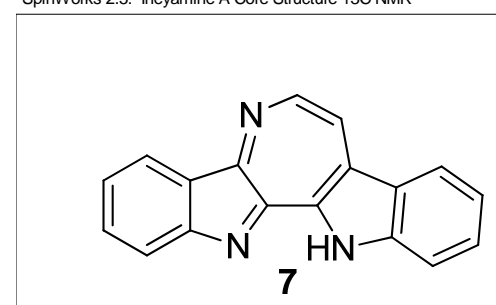

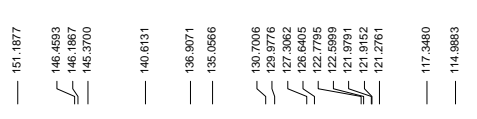

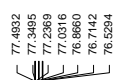

|

7

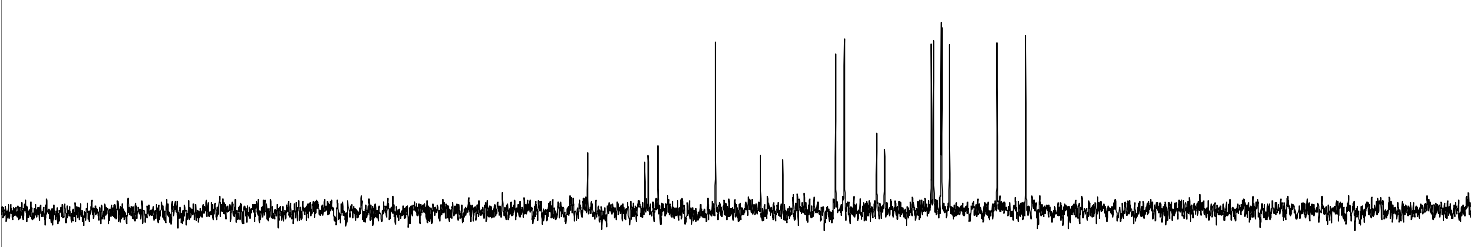

PPM

100.0

80.0

60.0

40.0

freq. of 0 ppm $100.547391 \mathrm{MHz}$
processed size: 32768 complex

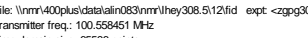

time domain size: 65536 points
width: $24038.46 \mathrm{~Hz}=239.049640 \mathrm{ppm}=0.366798 \mathrm{HE} / \mathrm{pt}$
number of scans: 3000

15 
SpinWorks 2.5: Bisindole trifluoroacetamide

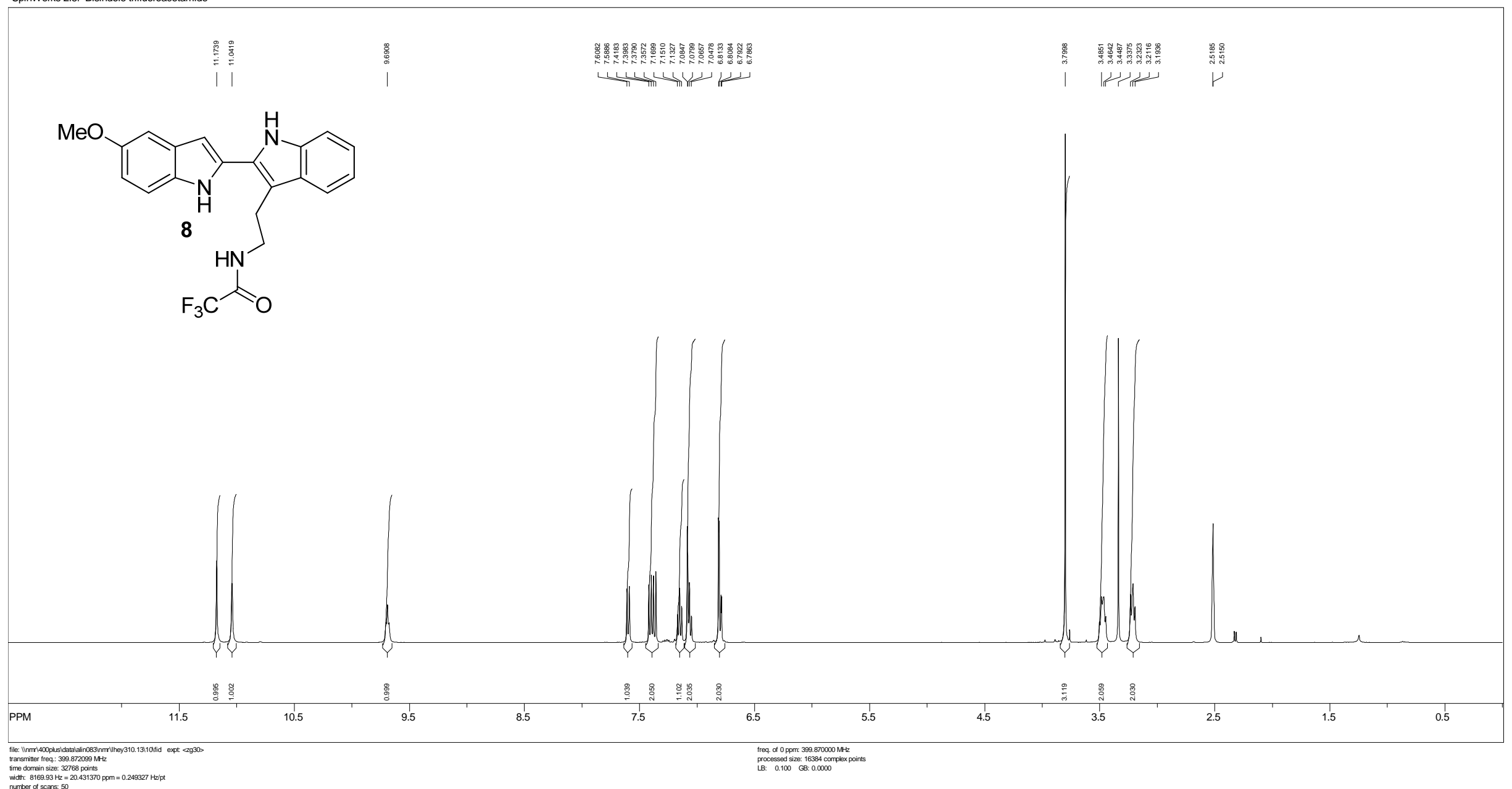


SpinWorks 2.5: Trifluoroacetamide bisindole 13C NMR

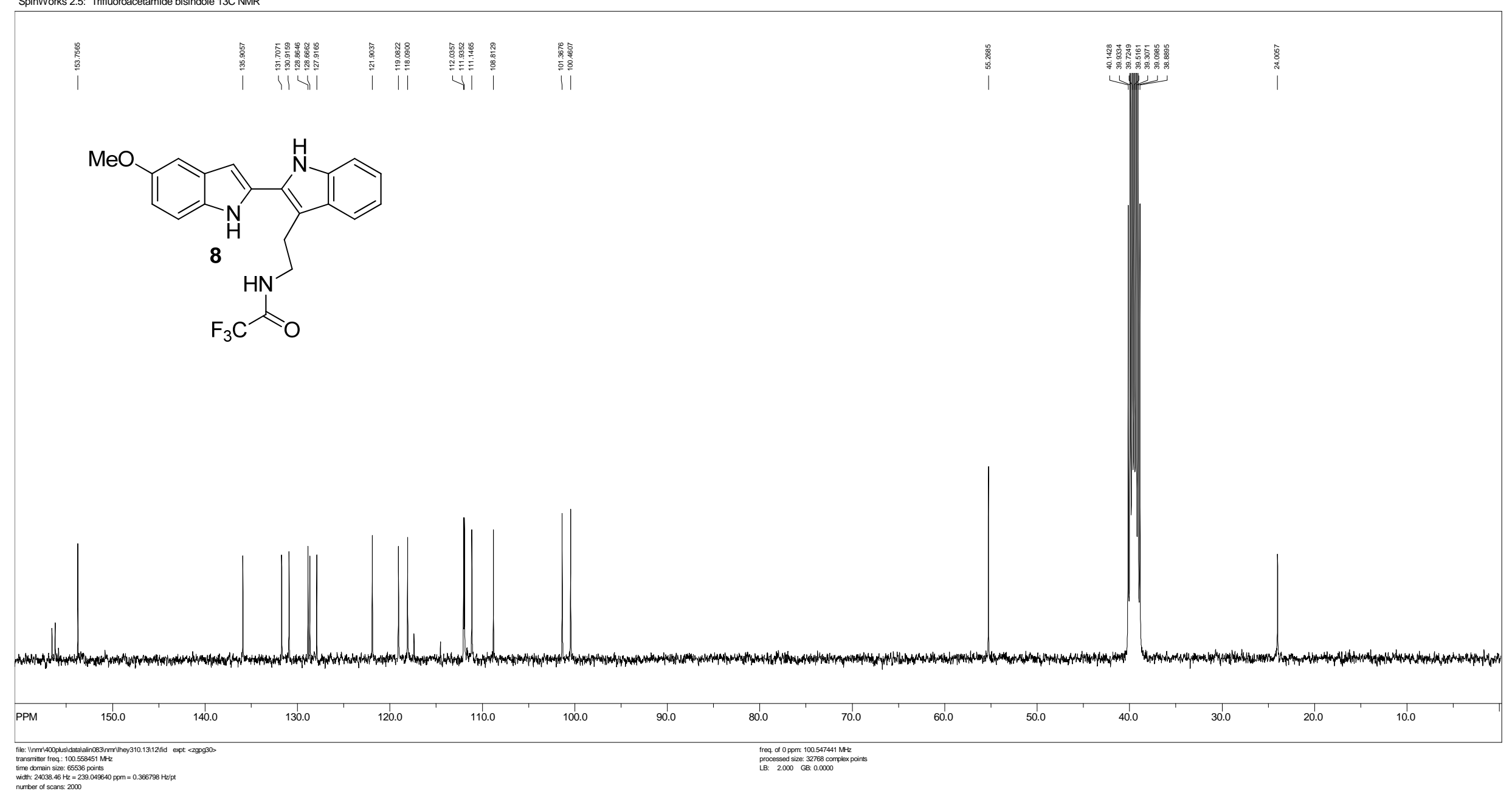


SpinWorks 2.5: Trifluoroacetamido bisindole HMBC

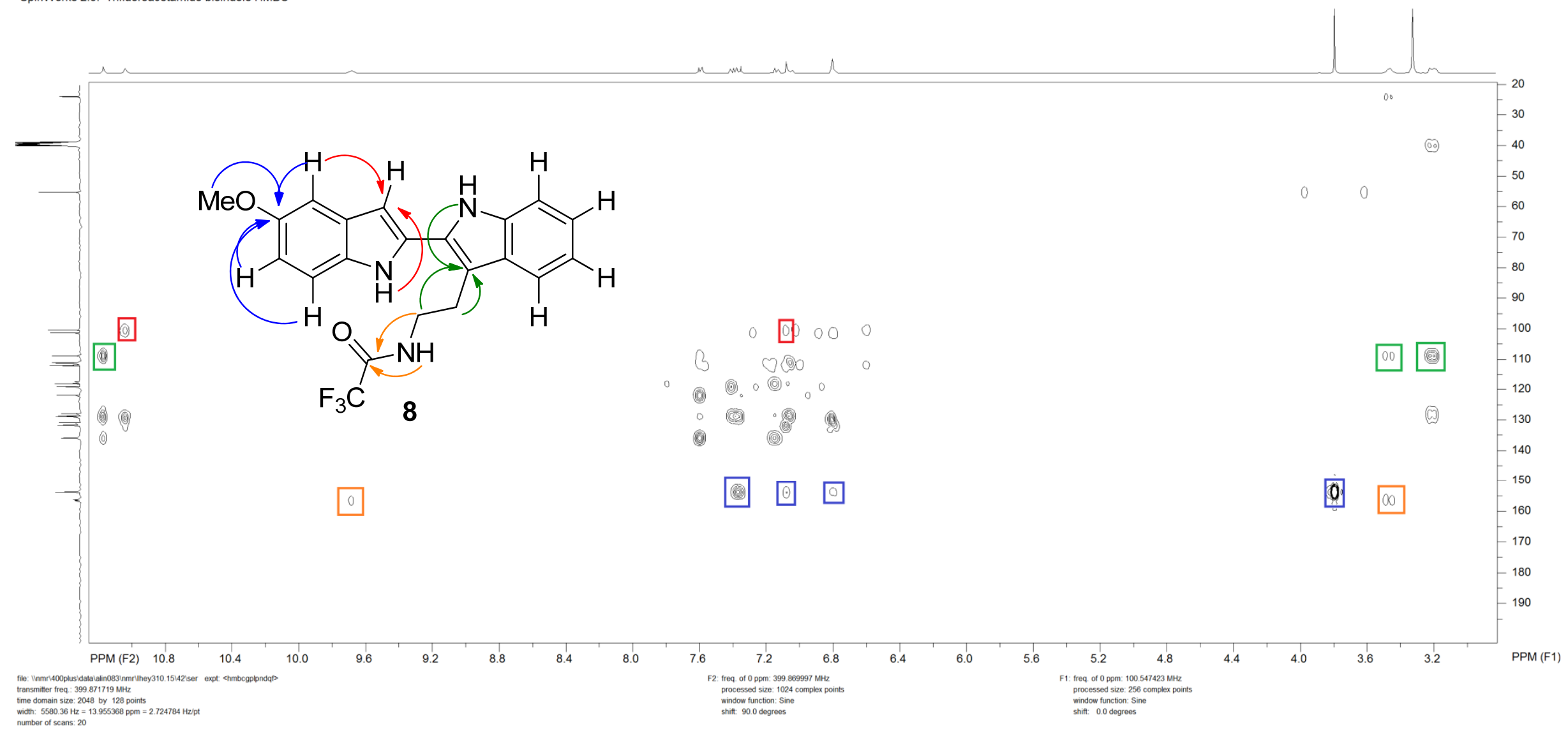




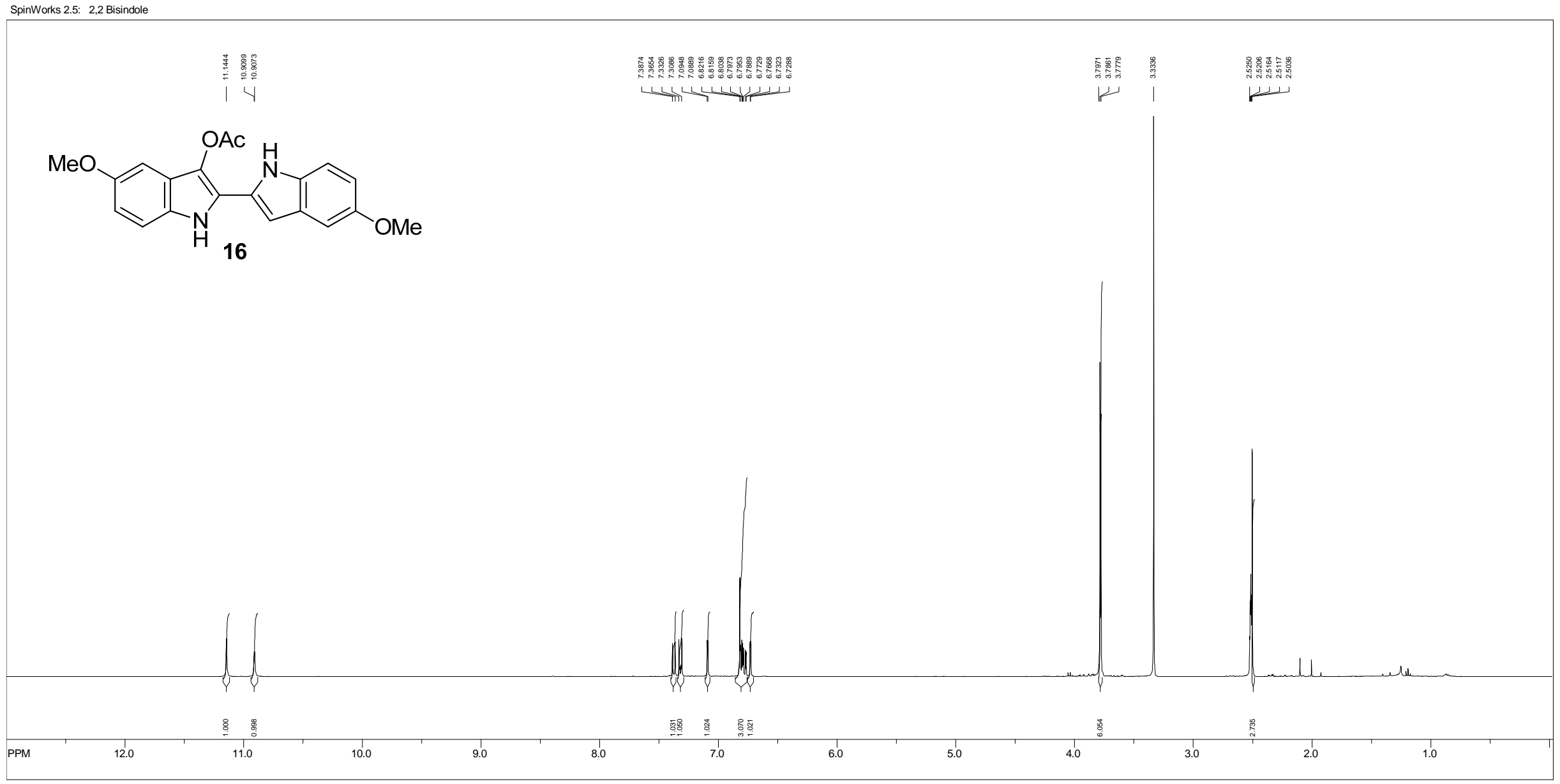

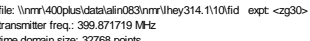

time domain size: 32768 points
width: $8169.93 \mathrm{~Hz}=20.431399 \mathrm{ppm}=0.249327 \mathrm{~Hz} / \mathrm{pt}$
number of $\mathrm{scans}: 16$

freq. of 0 ppr $399.870000 \mathrm{MHz}$
processed size: 16384 complex

LB: 0.000 GB: 0.0000

19 
SpinWorks 2.5: 2-2 Blsindole 13C NMR

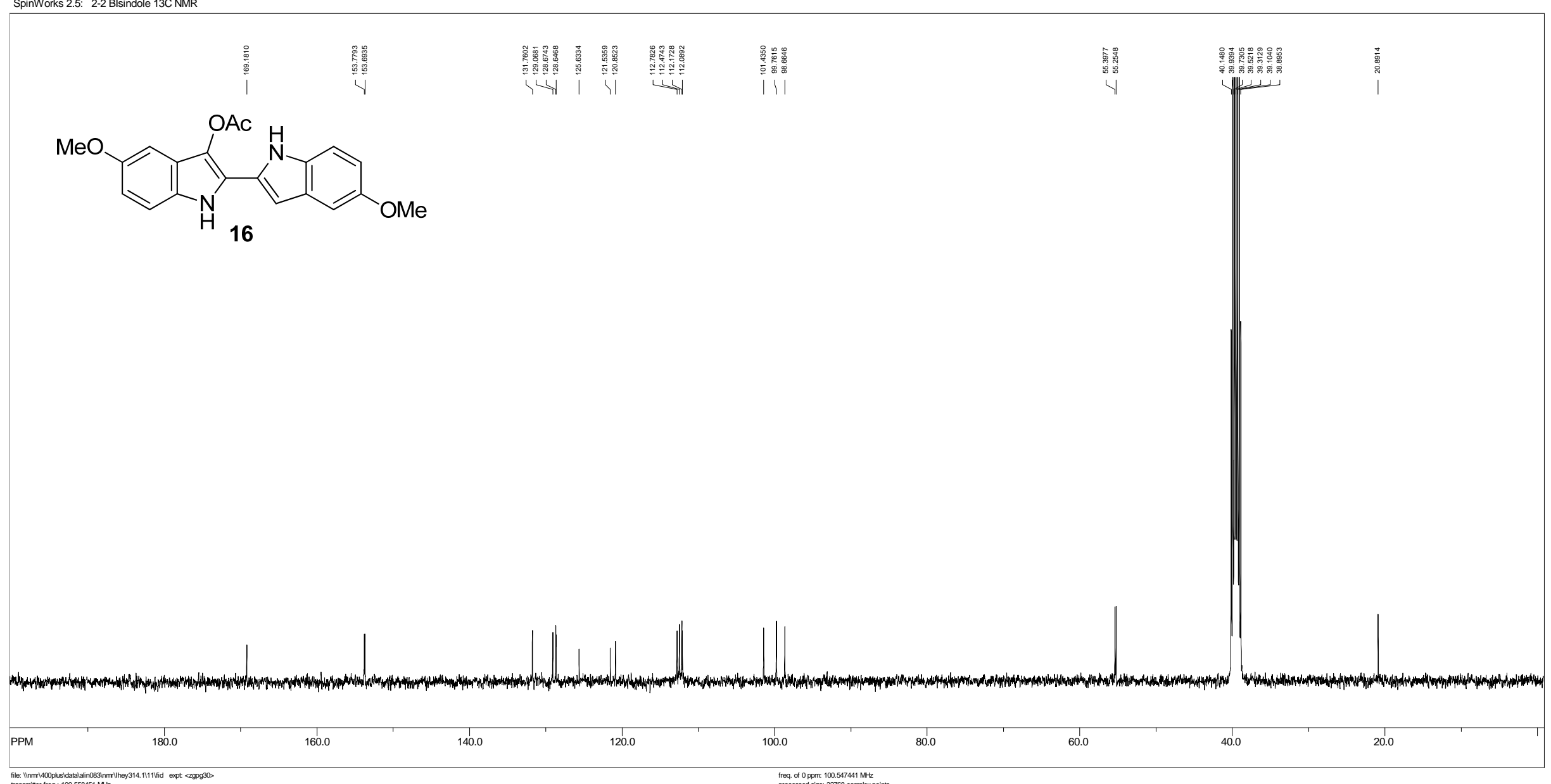

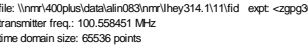

freq. of 0 ppmr $100.547441 \mathrm{MHz}$

une domain size: 650330 pants
number of scans: 1000

20 


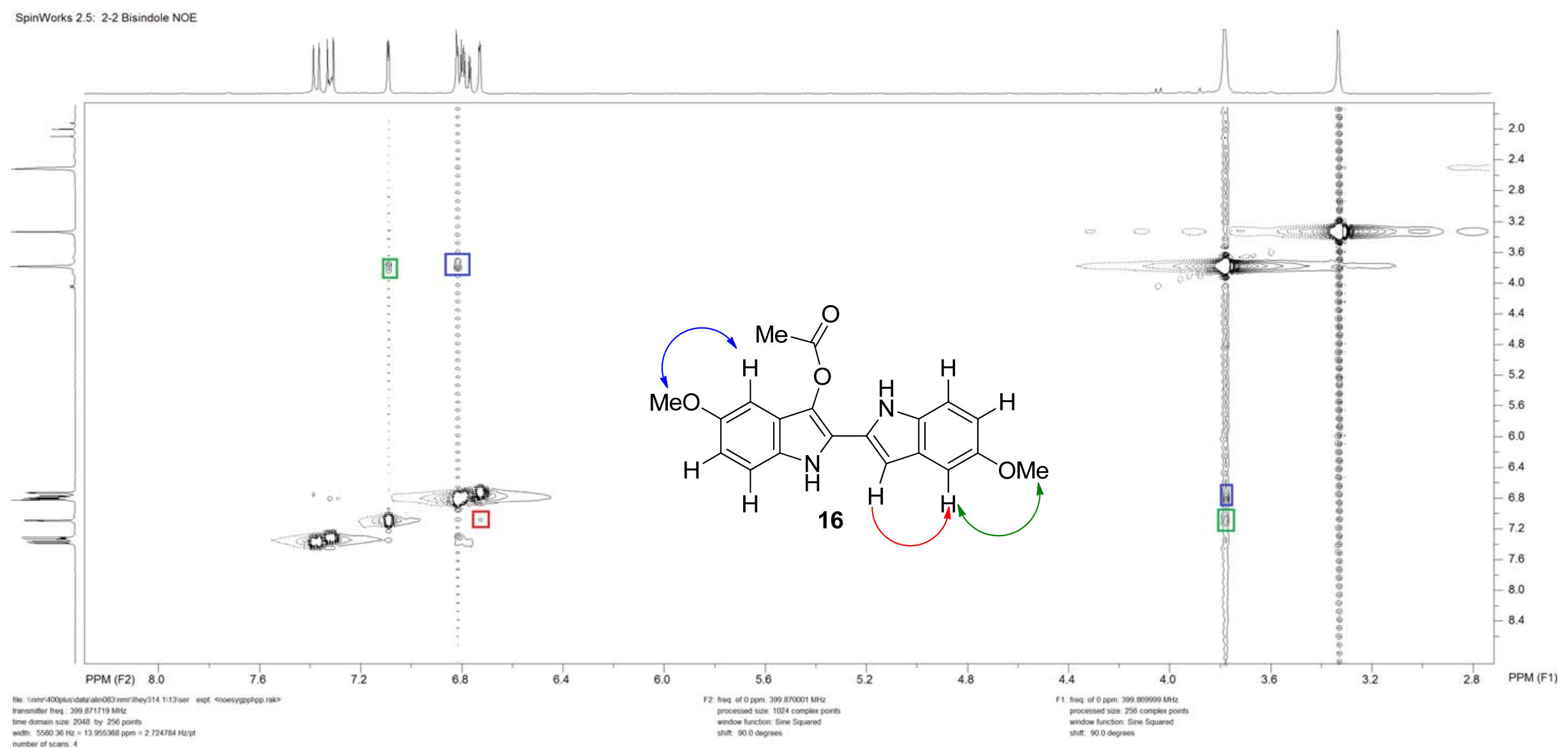


SpinWorks 2.5: protonstdri DMSO /nmr/400p alin083 39

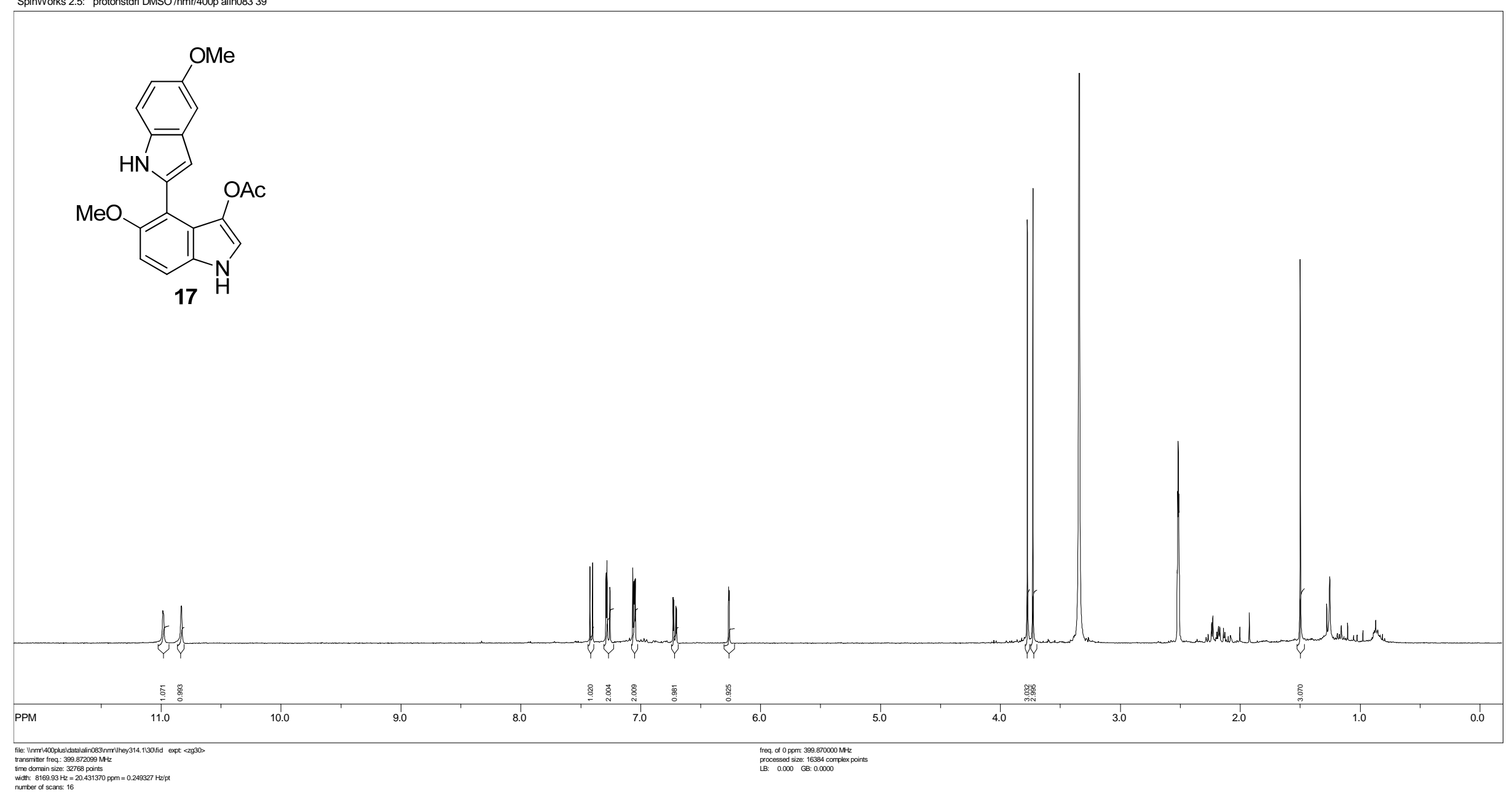




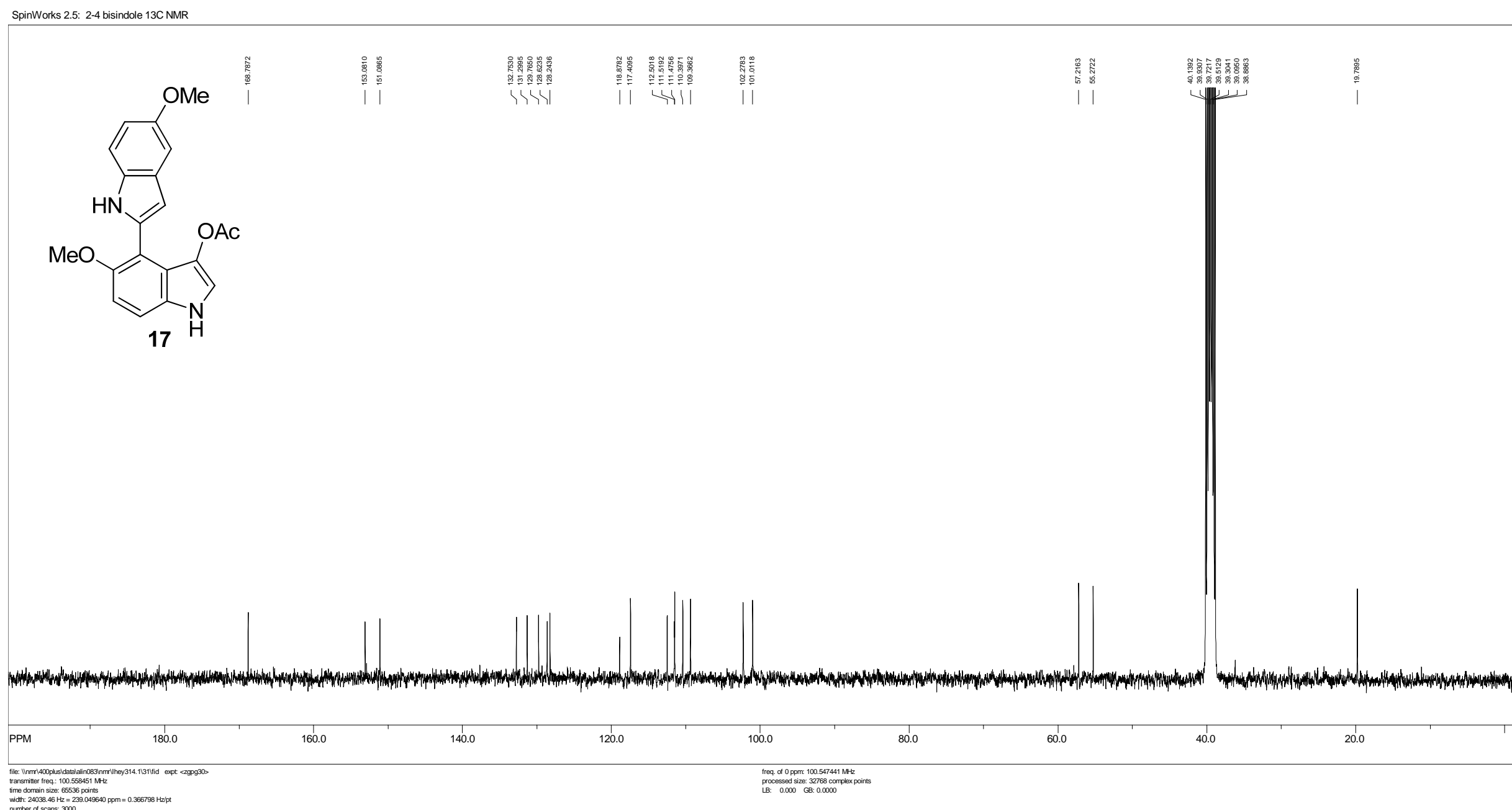


SpinWorks 2.5: 2.4 Bisindole NOE

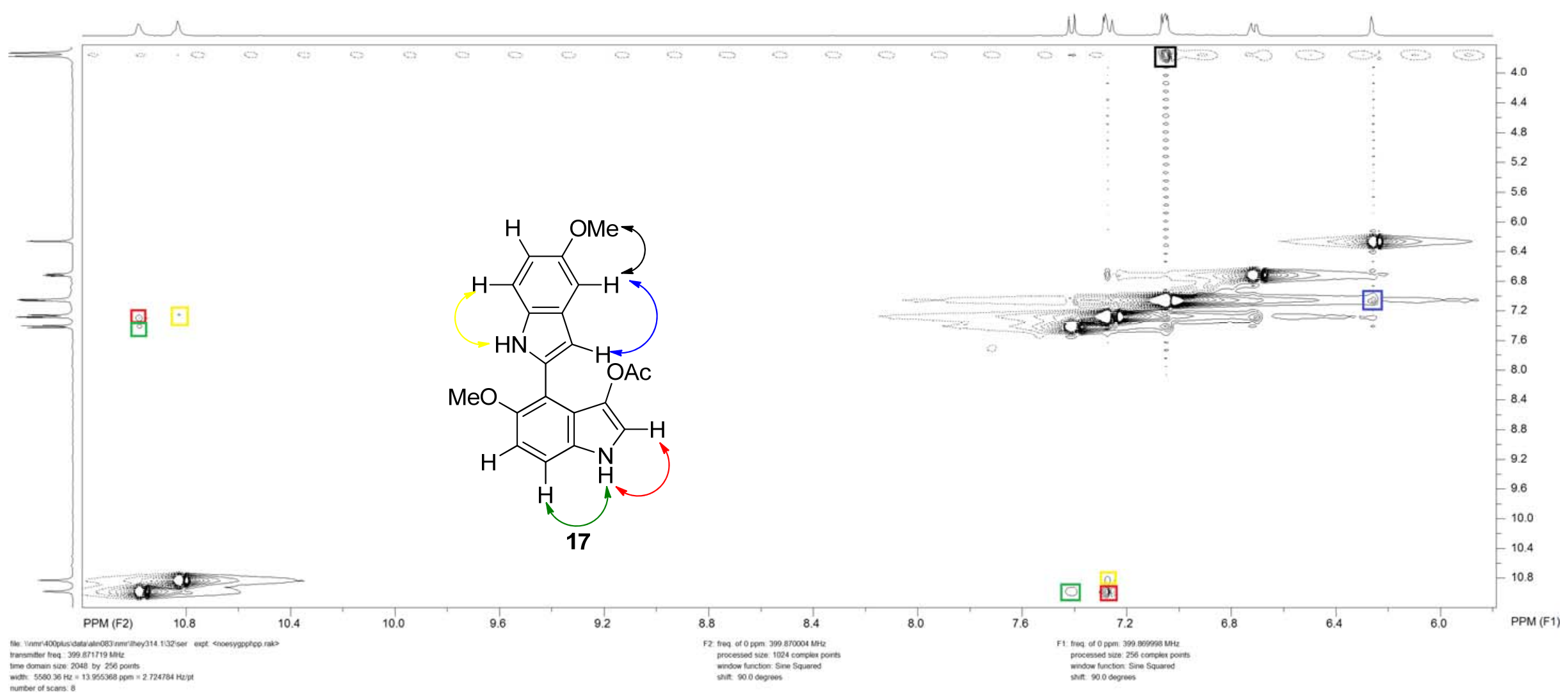


SpinWorks 2.5: Trifluoroacetamido Bisindolone

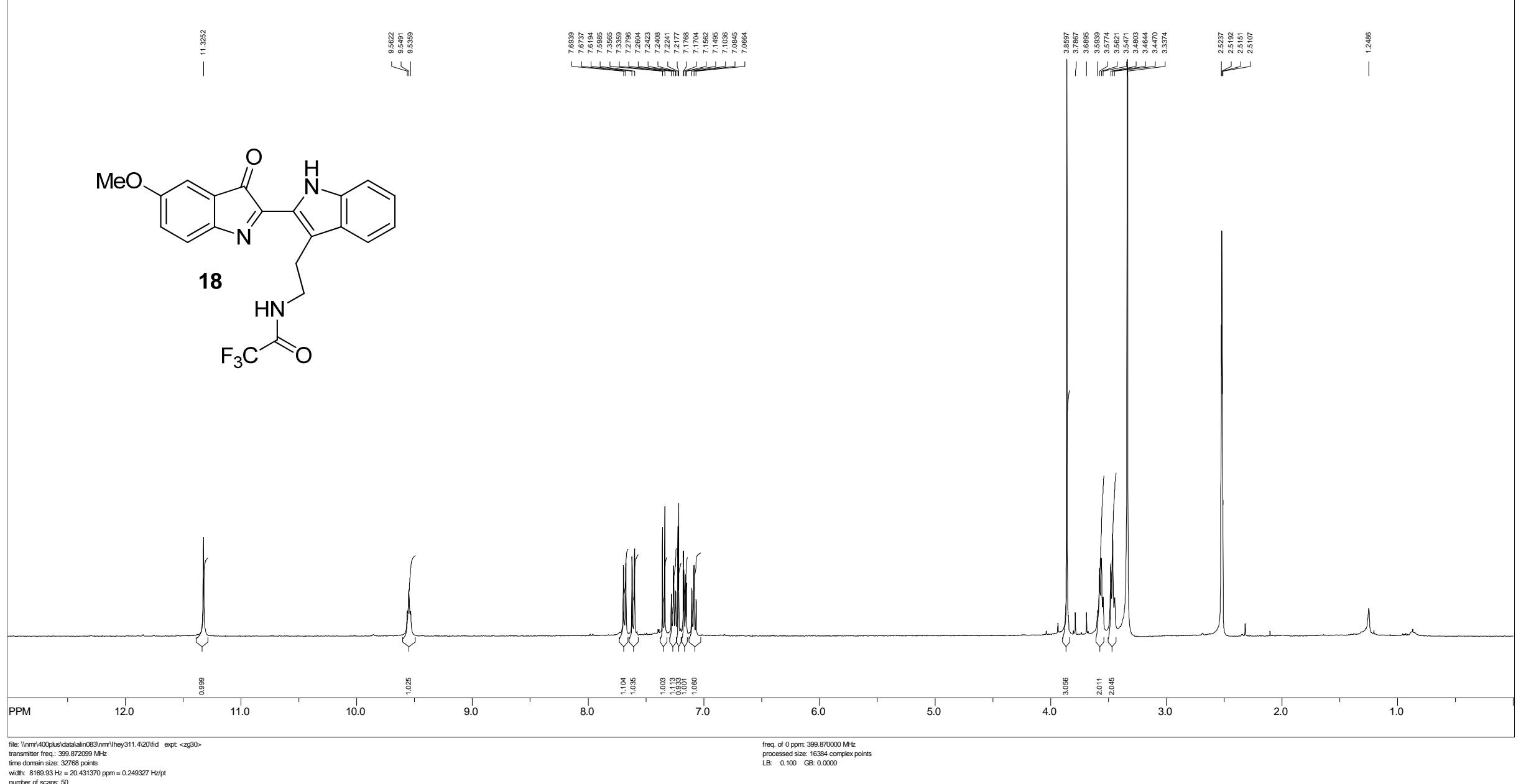




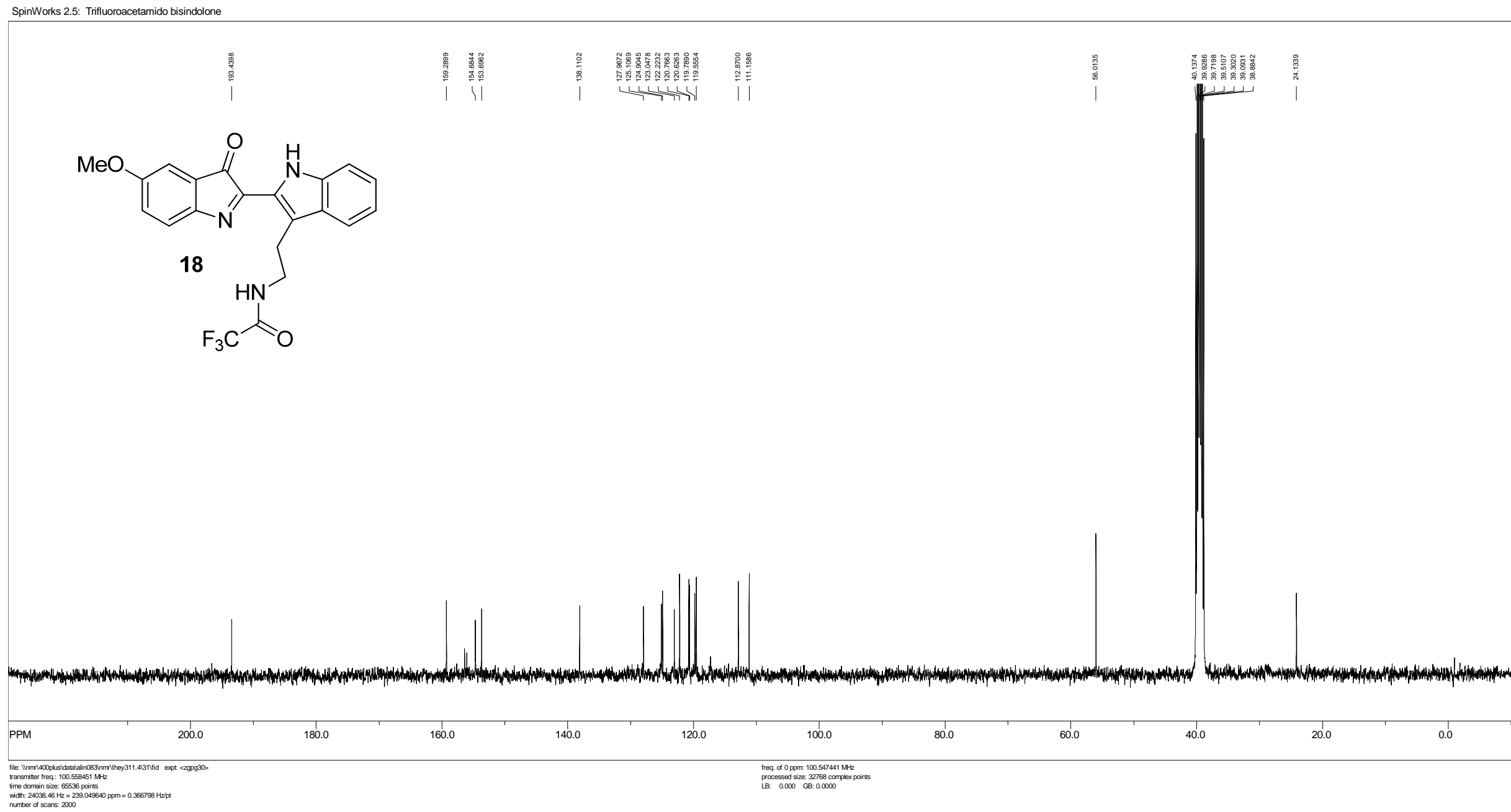


SpinWorks 2.5: Trifluoroacetamido indolone

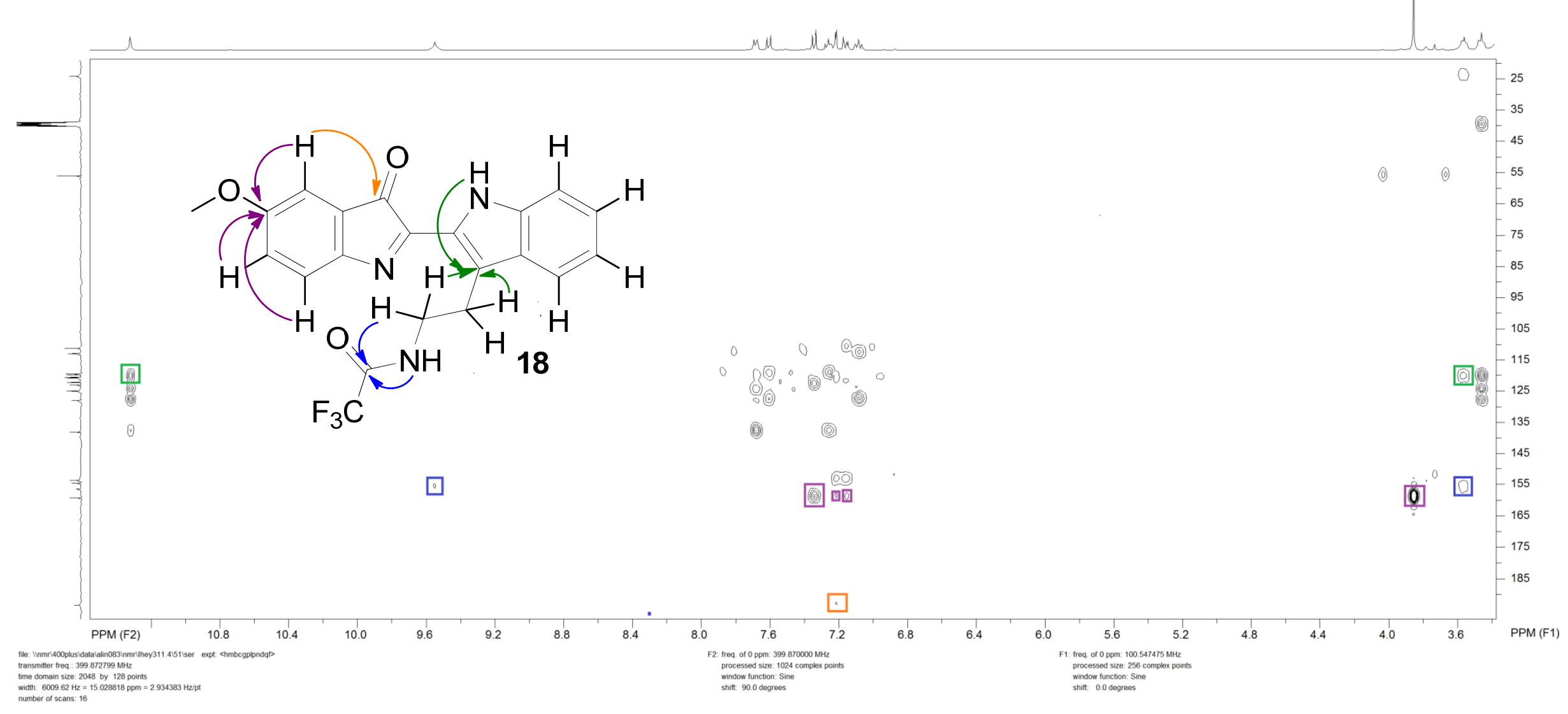


SpinWorks 2.5: protonstdri CDC13 /nmr/400p alin083 49

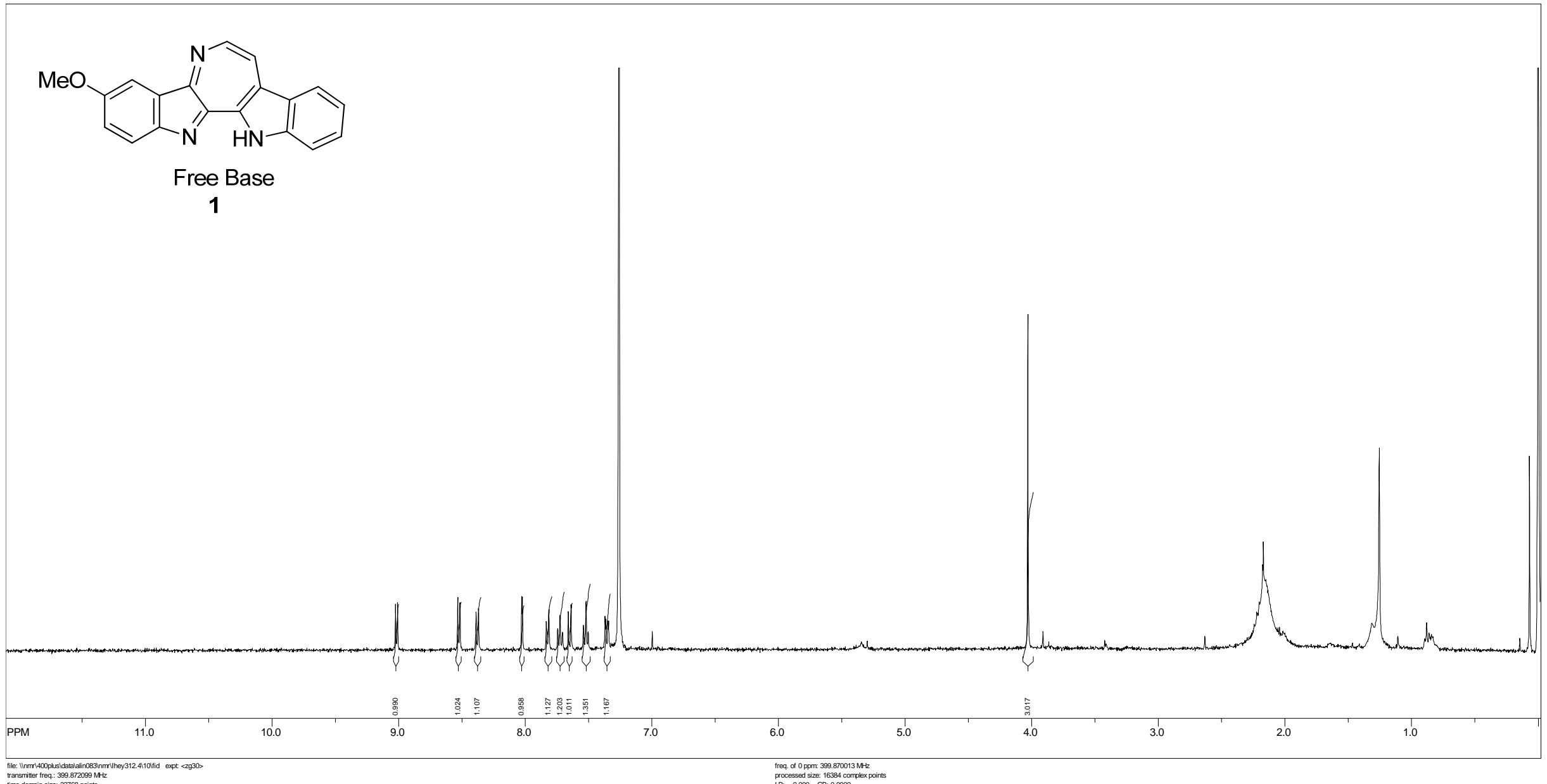




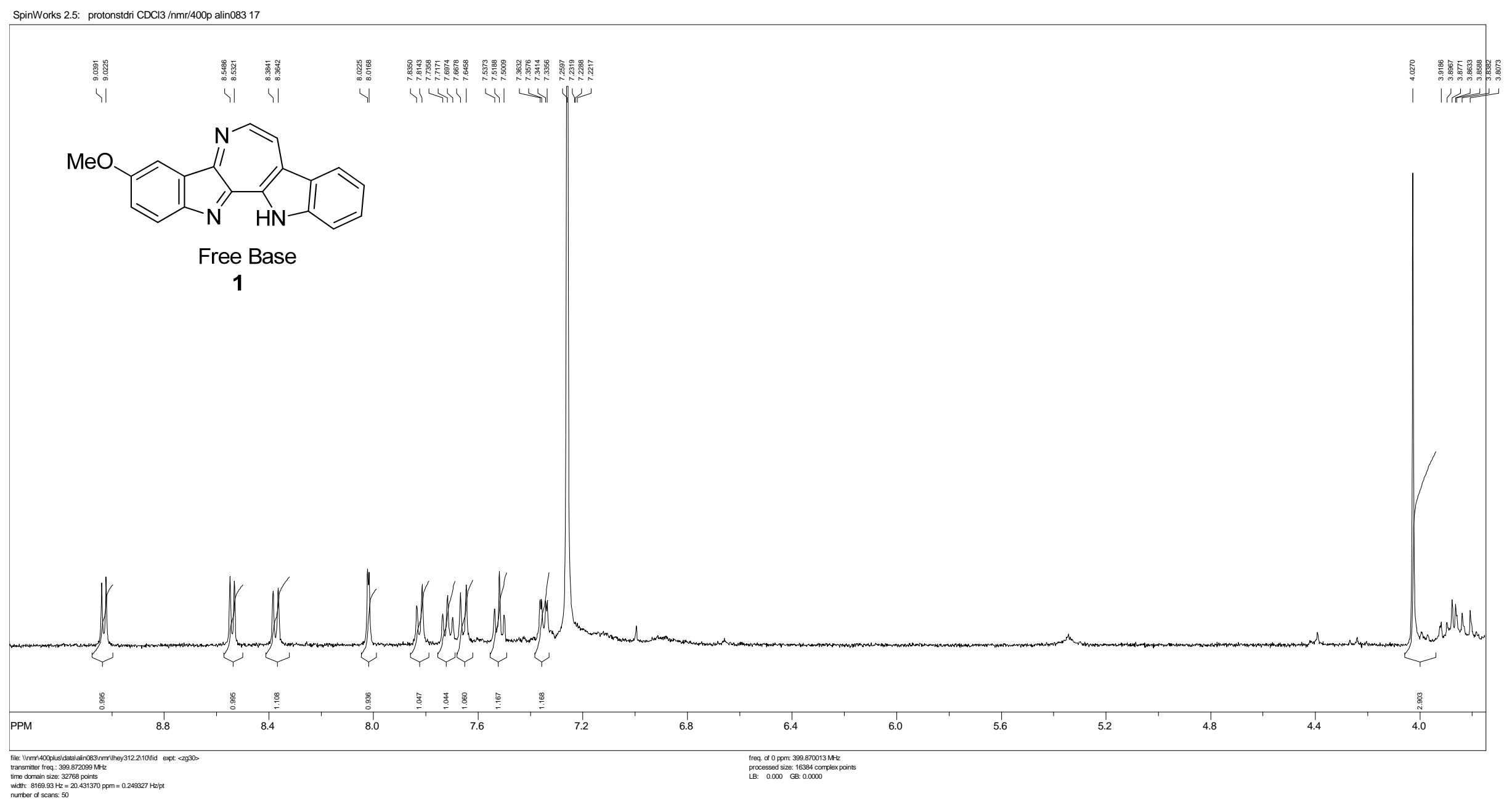




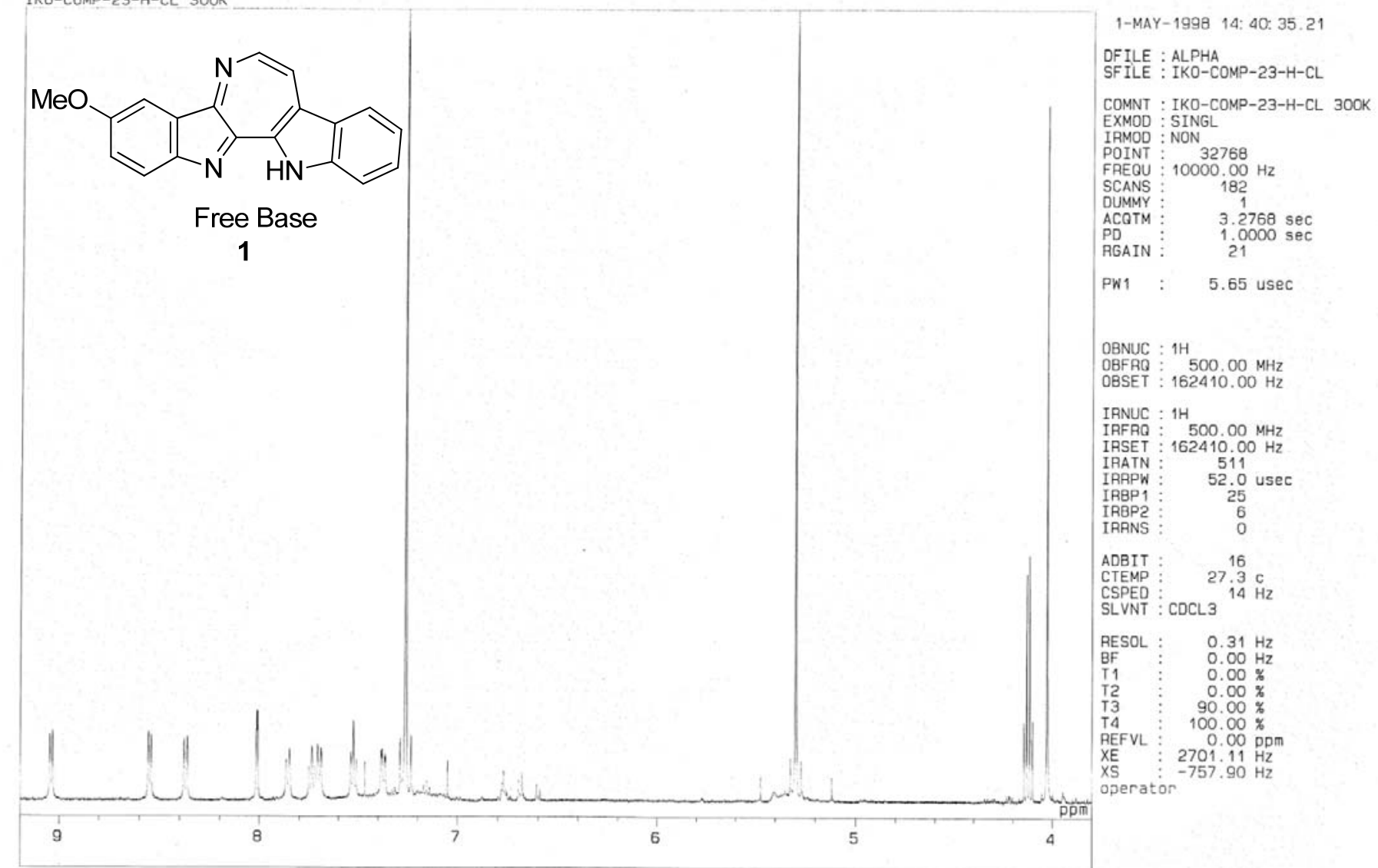

Spectrum provided by Professor Junichi Tanaka, Univeristy of the Ryukyus 
SpinWorks 2.5: Iheyamine A - TFA Salt

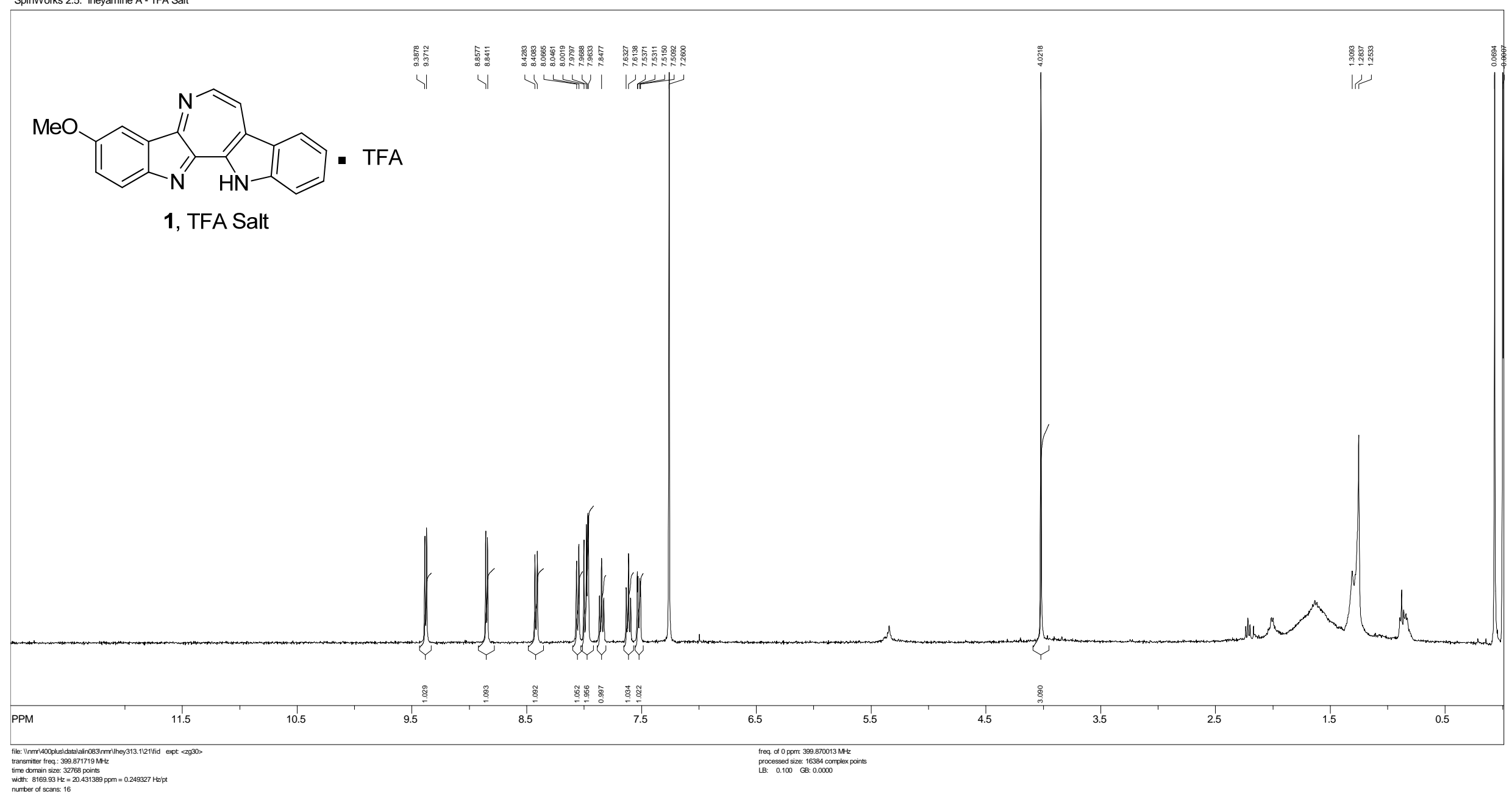


SpinWorks 2.5: Iheyamine A - TFA Salt

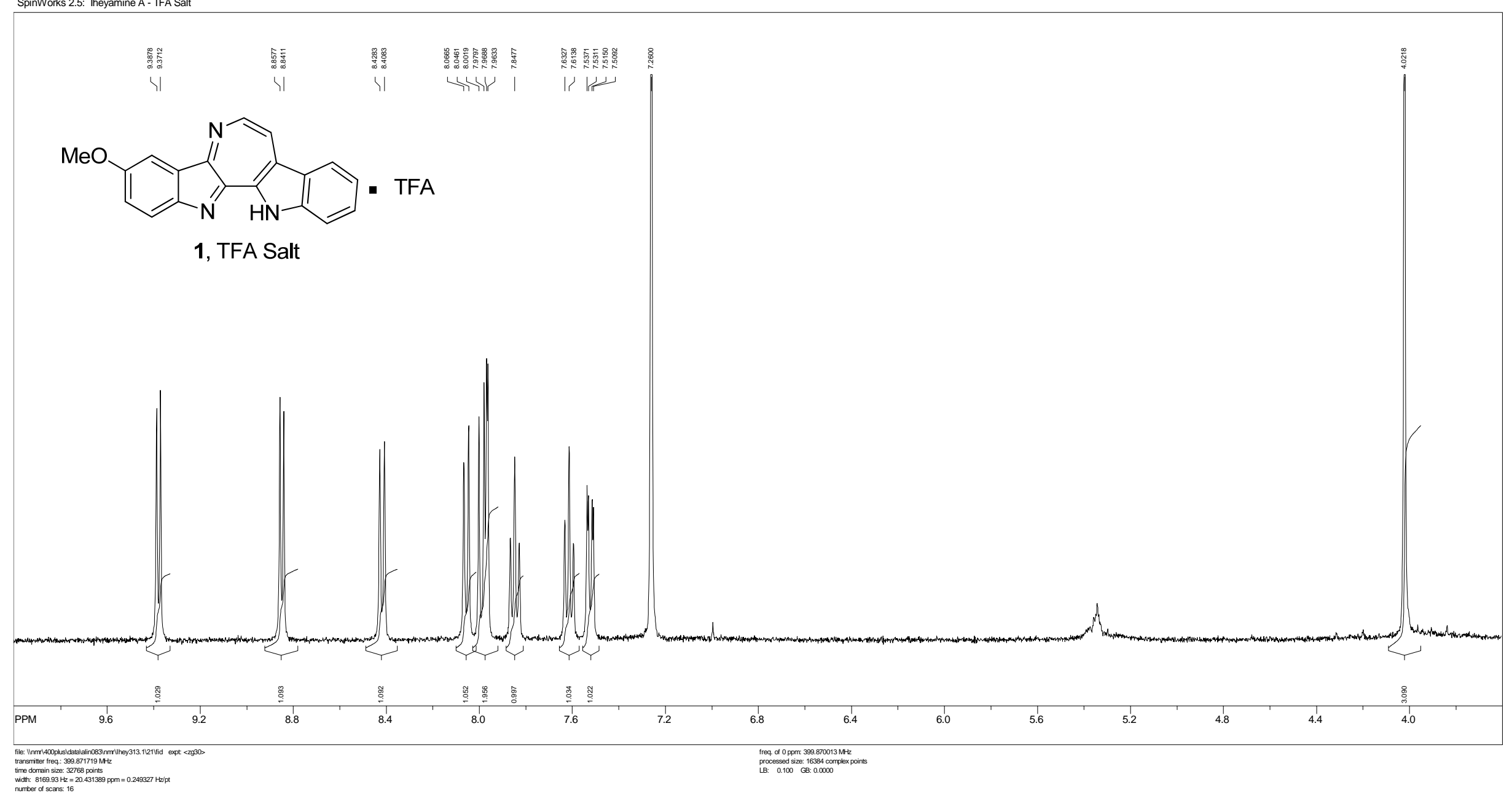




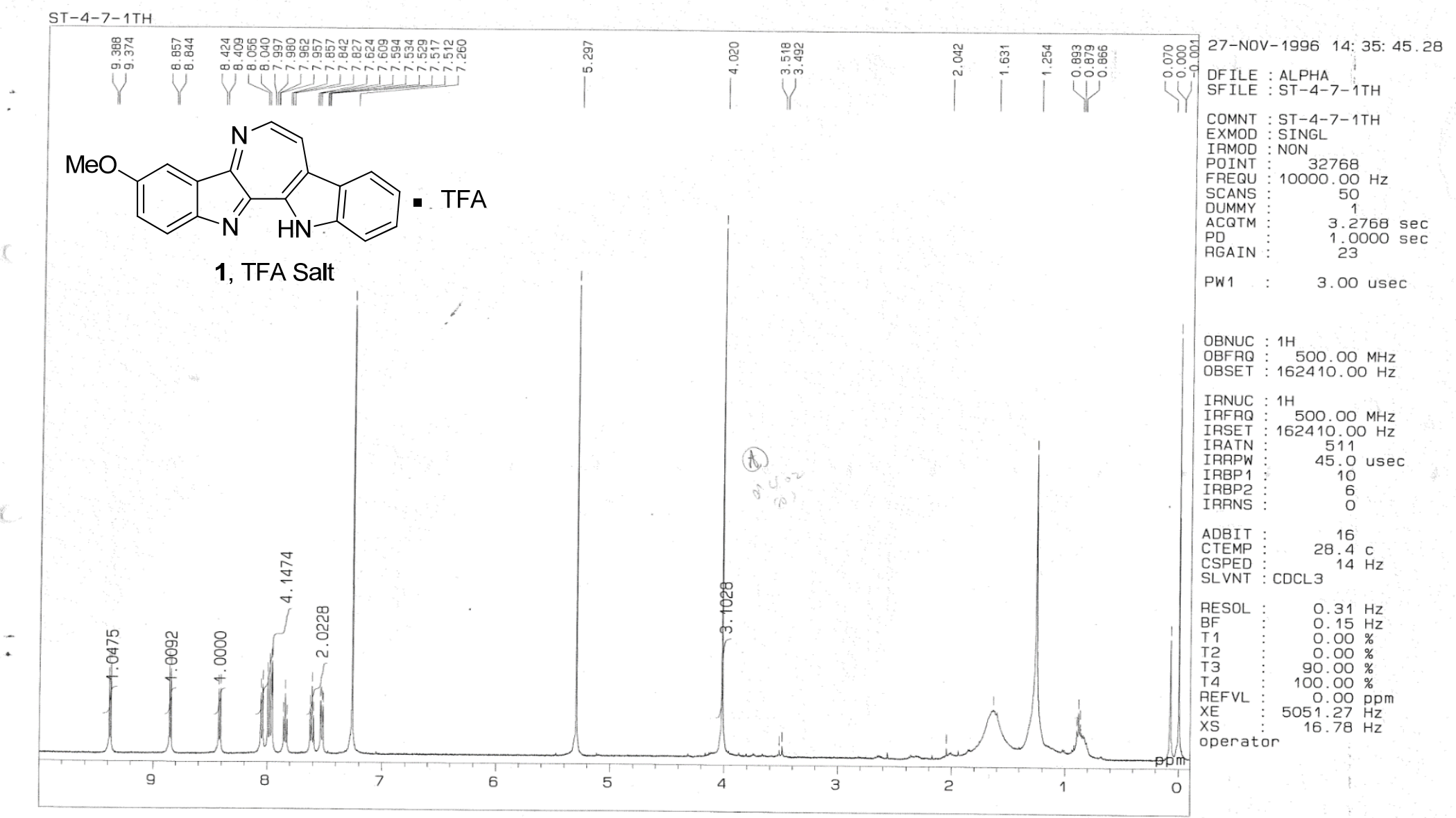

Spectrum provided by Professor Junichi Tanaka, University of the Ryukyus 


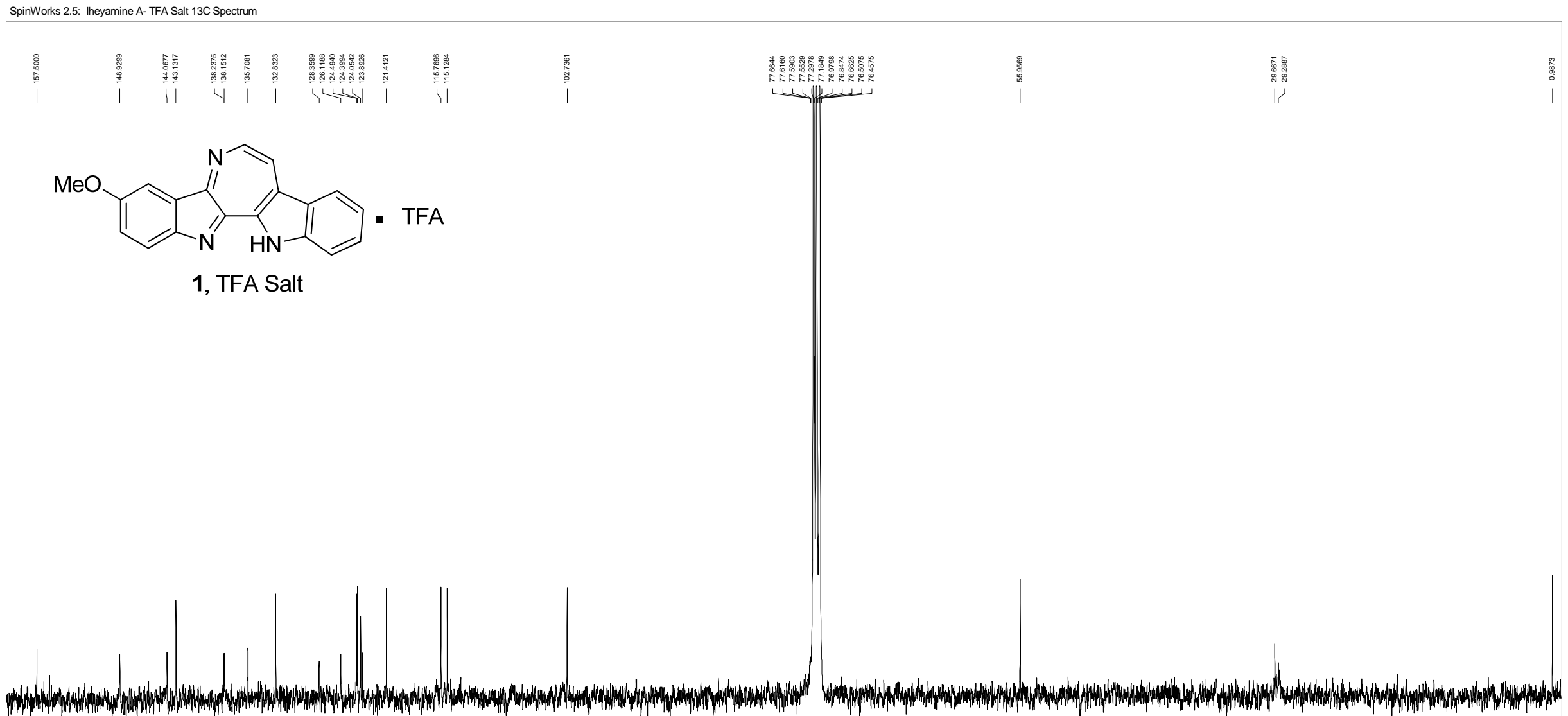

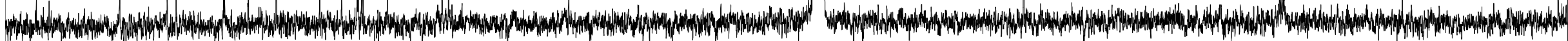

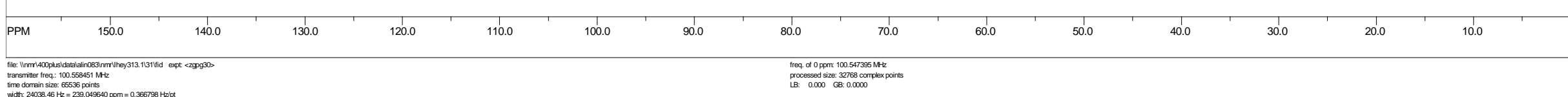




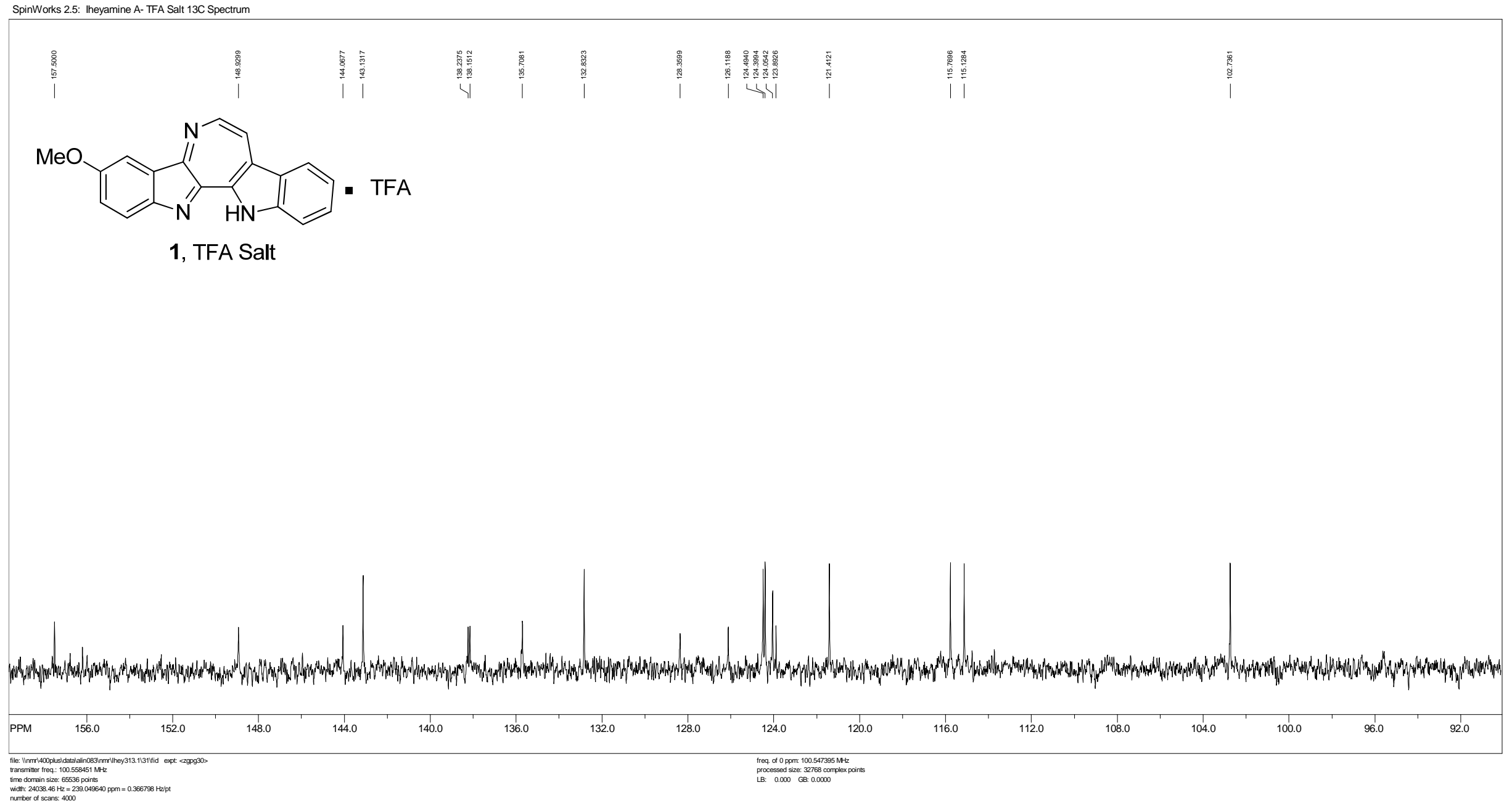

35 


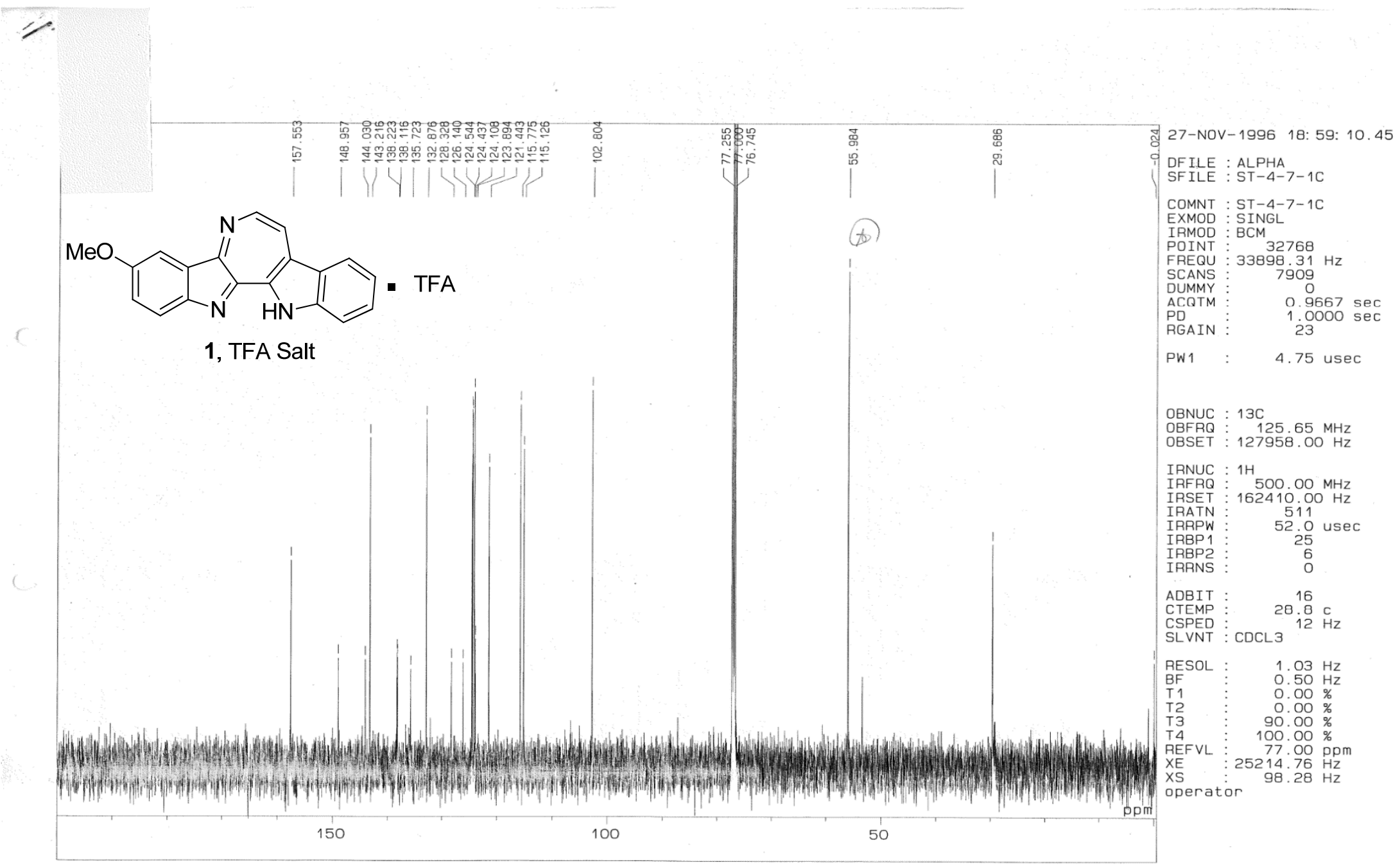

Spectrum provided by Professor Junichi Tanaka, University of the Ryukyus 


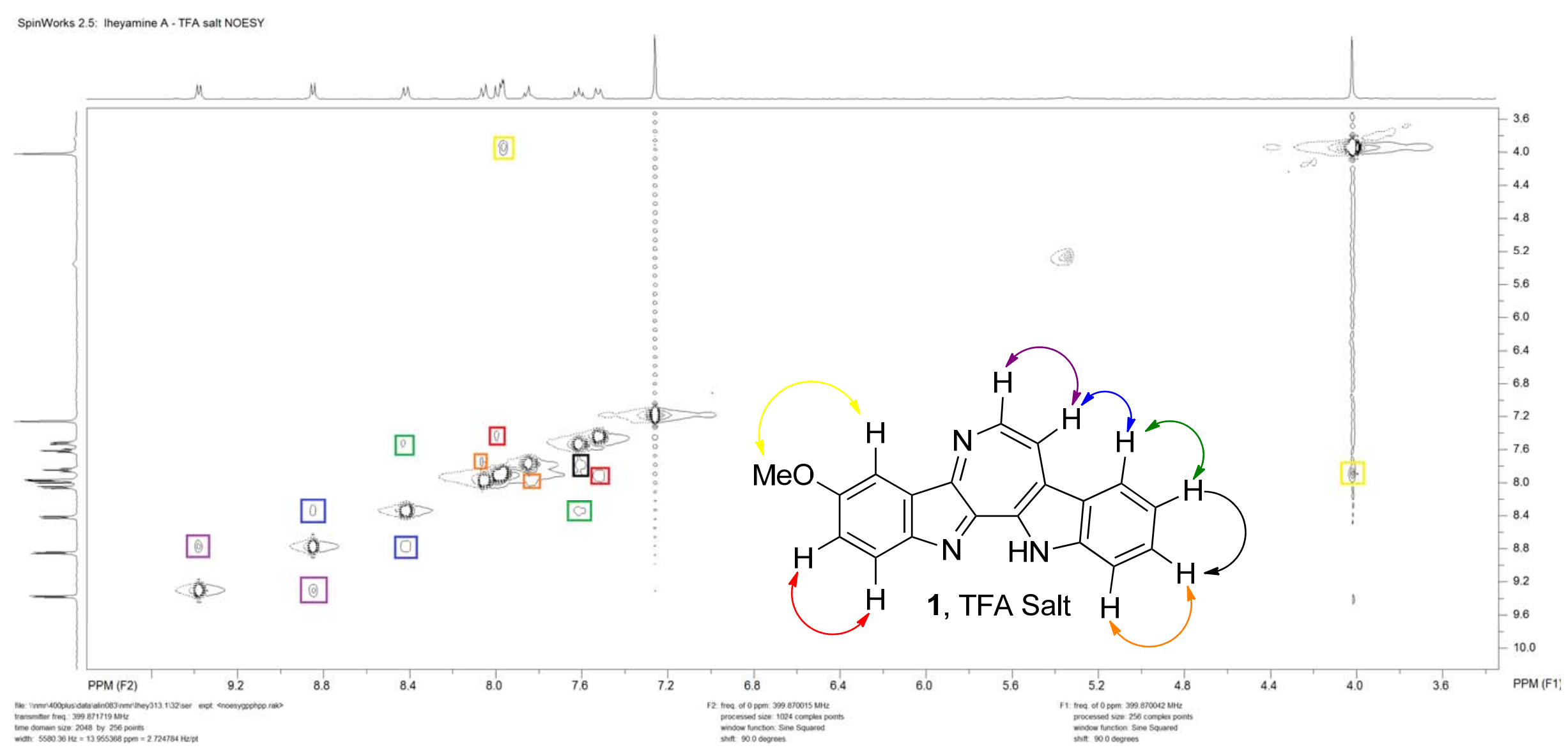



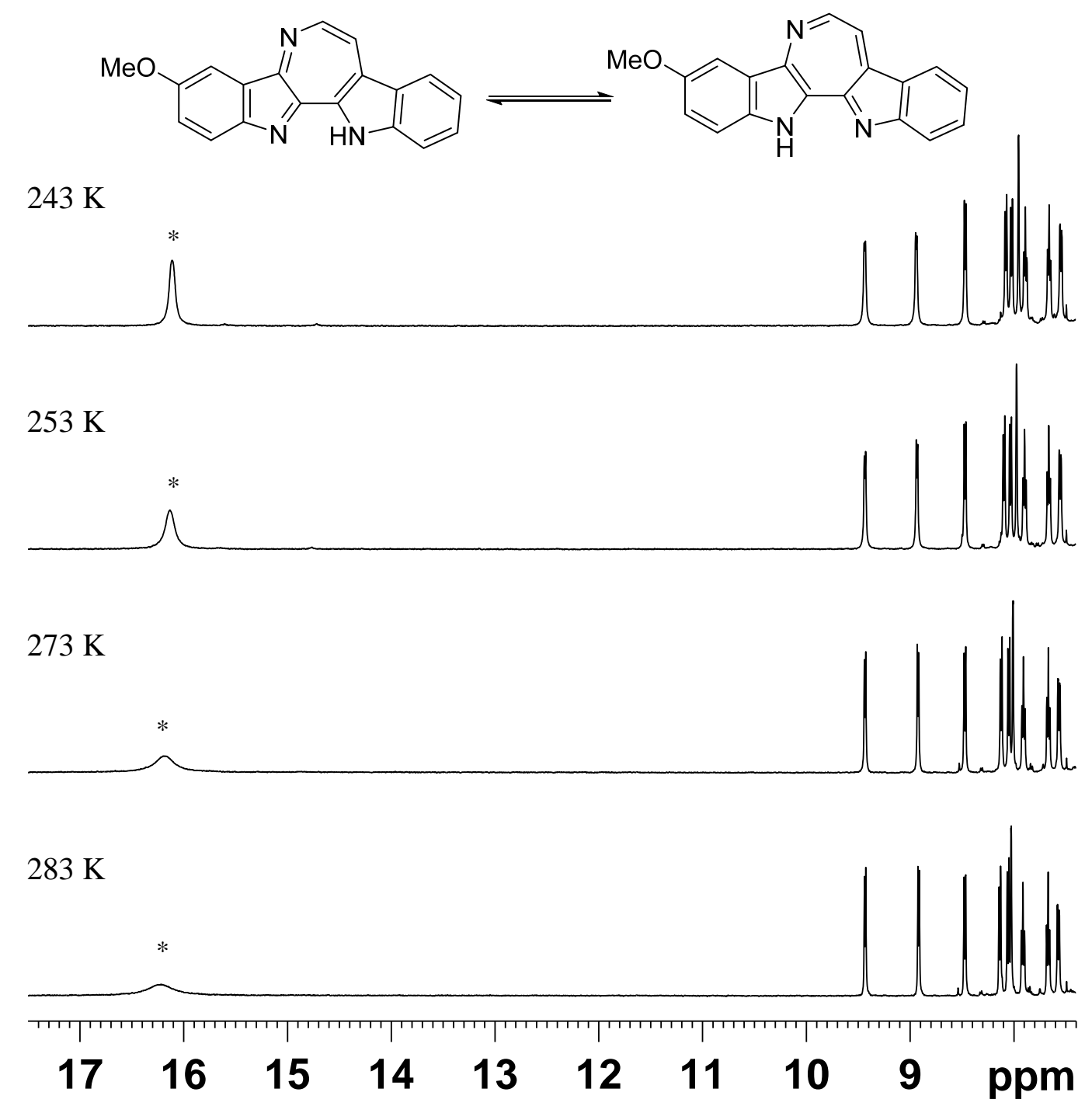

Variable temperature ${ }^{1} \mathrm{H}$ experiments of iheyamine (TFA salt) in $\mathrm{CDCl}$. TFA peak is indicated by an asterisk $\left({ }^{*}\right)$. Attempt to slow down $\mathrm{NH}$ exchange between the two tautomers at low temperature was unsuccessful. 